\title{
Non-periodic bifurcations of one-dimensional maps
}

\author{
VANDERLEI HORITA $\dagger$, NIVALDO MUNIZ $\ddagger$ and PAULO ROGÉRIO SABINI§ \\ $\dagger$ Departamento de Matemática, IBILCE/UNESP, Rua Cristóvão Colombo 2265, \\ 15054-000 S. J. Rio Preto, SP, Brazil \\ (e-mail: vhorita@ibilce.unesp.br) \\ $\ddagger$ Departamento de Matemática, UFMA, Avenida dos Portugueses, S/N, \\ 65000-000 São Luís, MA, Brazil \\ (e-mail:nmuniz@demat.ufma.br) \\ § Instituto de Matemática e Estatística, UERJ, Rua São Francisco Xavier 524, \\ 20550-900 Rio de Janeiro, RJ, Brazil \\ (e-mail: progerio@ime.uerj.br)
}

(Received 2 August 2004 and accepted in revised form 26 June 2006)

\begin{abstract}
We prove that a 'positive probability' subset of the boundary of '\{uniformly expanding circle transformations\}' consists of Kupka-Smale maps. More precisely, we construct an open class of two-parameter families of circle maps $\left(f_{a, \theta}\right)_{a, \theta}$ such that, for a positive Lebesgue measure subset of values of $a$, the family $\left(f_{a, \theta}\right)_{\theta}$ crosses the boundary of the uniformly expanding domain at a map for which all periodic points are hyperbolic (expanding) and no critical point is pre-periodic. Furthermore, these maps admit an absolutely continuous invariant measure. We also provide information about the geometry of the boundary of the set of hyperbolic maps.
\end{abstract}

\section{Introduction}

We begin by formulating the fundamental problem motivating this work and stating our main results.

1.1. Non-periodic bifurcation. A great deal of understanding of those dynamical systems lying beyond the hyperbolic (Axiom A [26]) domain has been achieved through the following strategy. One considers parametrized families $\left(\Phi_{\theta}\right)_{\theta \in[-1,1]}$ of systems (diffeomorphisms or flows) such that $\Phi_{\theta}$ is hyperbolic for initial $\theta=-1$, but crosses the boundary of hyperbolic systems at some parameter, say $\theta=0$. Then one tries to understand the typical behavior of $\Phi_{\theta}$, for small $\theta>0$. 
This problem is probably not realistic without some further assumptions about the dynamics at $\theta=0$. In a series of papers that appeared in the 1970s and the early 1980s, Newhouse, Palis and Takens were able to develop a detailed theory of the case when the limit set (forward and backward accumulation points of all orbits) of $\Phi_{0}$ is finite; see [16-19]. In this case, for a generic (Baire second category) set of arcs of diffeomorphisms, the first bifurcation corresponds to one of the following two situations.

(NH) There exists a unique periodic orbit that is non-hyperbolic, and it is either a saddlenode (one eigenvalue equal to 1 ), a period-doubling (one eigenvalue equal to -1 ), or a Hopf orbit (two complex conjugate eigenvalues with norm 1).

(NT) All the periodic orbits are hyperbolic, but there exists a unique non-transverse intersection between some stable and some unstable manifold of periodic orbits; this intersection is quasi-transverse (codimension 1$)$.

These two bifurcation mechanisms (NH) and (NT) may be responsible for drastic changes of the global dynamics, which have been extensively studied by many authors, for both finite and infinite limit set. See [5, 6, 14, 20-24, 27], among many other works. See also $[\mathbf{9}, \mathbf{1 0}]$ for related results about certain classes of maps arising from vector fields. Moreover, Newhouse and Palis [17] conjectured that the first bifurcation of generic families starting from a Morse-Smale system (i.e. with no assumption on the limit set at the bifurcation parameter) is either (NH) or (NT). However, to the best of our knowledge there has been no progress in the direction of this conjecture.

In fact, our results in this work provide a negative answer to a natural probabilistic reformulation of the conjecture for one-dimensional maps. That is, we prove that there exist large (in a measure theoretical sense) subsets of the boundary of the hyperbolic domain constituted by maps whose periodic orbits are all hyperbolic and whose critical points are not pre-periodic. Let us point out that pre-periodic critical points play the role of homoclinic tangencies in the context of one-dimensional maps. The reason hyperbolicity breaks down for our maps is the presence of some non-periodic cubic critical point (see §1.4).

More precisely, our main result is the following.

THEOREM A. There exists an open set $\mathcal{U}$ of two-parameter families $\left(f_{a, \theta}\right)_{a, \theta}$ of maps of the circle such that, for some $\theta_{*}=\theta_{*}(a), f_{a, \theta_{*}}$ has a (cubic) critical point (and so cannot be uniformly expanding). Moreover, for a positive Lebesgue measure set $\mathcal{A}$ of parameters $a$ :

(1) $f_{a, \theta_{*}}$ belongs to the boundary of the uniformly expanding domain; and

(2) all periodic points of $f_{a, \theta_{*}}$ are hyperbolic (expanding), and no critical point is preperiodic.

In other words, a 'positive probability' subset of the boundary of hyperbolic systems consists of Kupka-Smale maps. In fact, in this setting the Lyapunov exponents at periodic points are uniformly bounded away from zero (see $§ 7$ ).

An interesting related question is whether $f_{a, \theta_{*}}$ belongs to the boundary of some connected component of the uniformly hyperbolic domains: while this does not follow immediately from our arguments, numerical evidence suggests that this is probably the case. Indeed, it seems that there are continuous curves in parameter space starting at $f_{a, \theta_{*}}$ 
for each $a \in \mathcal{A}$, and otherwise consisting of uniformly expanding maps. A stronger statement would be that $f_{a, \theta}$ is uniformly expanding for every $\theta<\theta_{*}$ close to $\theta_{*}$, but computer evidence does not seem to support this.

For dimension two, Sabini, in [25], exhibits dissipative diffeomorphisms with nonperiodic bifurcation: all periodic orbits are hyperbolic, their stable and unstable manifolds intersect transversally, but there is a large (in a measure theoretical sense) set of diffeomorphisms with cubic tangencies between stable and unstable manifolds, one of them associated to a non-periodic orbit. The one-dimensional setting we treat here, and which was not covered previously, is particularly interesting because it displays many features in a more transparent way, besides allowing for sharper arguments and conclusions. In fact, the methods we develop here lead to an improved version of the results in [25], which will appear in a joint paper by the present three authors.

1.2. Absolutely continuous invariant measures. Having proved abundance of nonperiodic bifurcations, in Theorem A, one would like to say more about these systems, namely, one would like to describe their dynamics at (and, at a second stage, beyond) the bifurcation.

Our next result is a contribution in this direction: we prove that the maps $f_{a, \theta_{*}}$ have well-defined asymptotics at the statistical level. More precisely, we have the following result.

THEOREM B. For every family $\left(f_{a, \theta}\right)_{a, \theta} \in \mathcal{U}$ with $a \in \mathcal{A}$ there exists a unique absolutely continuous invariant measure for $f_{a, \theta_{*}}$. This measure $\mu$ is supported in all $\mathbb{S}^{1}$ and is, in fact, a physical (SRB) measure: the time average $n^{-1} \sum_{j=0}^{n-1} \varphi\left(f_{a, \theta}^{j}(x)\right)$ converges to the space average $\int \varphi d \mu$ for Lebesgue almost every $x \in \mathbb{S}^{1}$ and every continuous function $\varphi$.

This statement should be related to the remarkable work of Jakobson [8], where he showed that the quadratic family has an absolutely continuous invariant measure for a positive measure set of parameters; see also [2, 4]. More recently, Lyubich [12] and Avila and Moreira [1] have shown that, for generic families of unimodal maps, for almost every parameter either there exists an absolutely continuous invariant measure or the map is hyperbolic (the critical point is in the basin of a periodic attractor). Moreover, the second alternative corresponds to an open and dense set of parameters, by Lyubich [11] and Graczyk and Swiatek [7]. Motivated by these results, we propose two problems.

Problem 1. Does $(\mathrm{NH}) \cup(\mathrm{NT})$ contain an open and dense subset of the boundary of expanding maps of the circle?

Problem 2. Has $(\mathrm{NH}) \cup(\mathrm{NT}) \cup$ \{maps with a unique critical (cubic) point \} full probability in the boundary?

1.3. On the boundary of hyperbolic maps. Given a two-parameter family $\left(f_{a, \theta}\right)_{a, \theta} \in \mathcal{U}$ we can induce a set $\mathcal{U}^{\prime}$ of one-parameter families $\left(f_{a, \theta}\right)_{\theta}$. A large set of families in $\mathcal{U}^{\prime}$ cross the boundary of hyperbolic maps at $\theta=\theta_{*}$, namely families $\left(f_{a, \theta}\right)_{\theta}$ with $a$ in $\mathcal{A}$. On the other hand we can find several families in $\mathcal{U}^{\prime}$ which leave the set of hyperbolic maps strictly before $\theta_{*}$, i.e. before the critical point is created, as stated in the next theorem. 


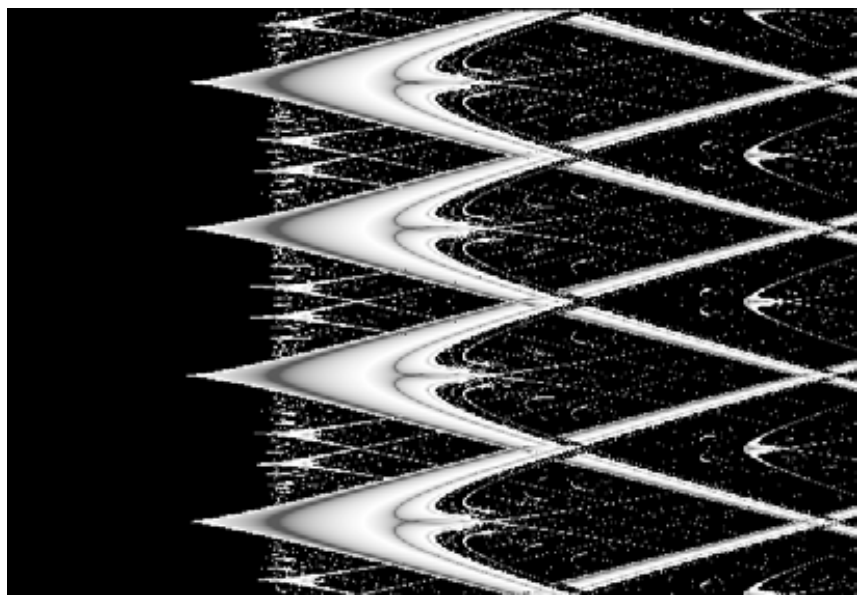

FIGURE 1. Boundary of hyperbolic maps.

THEOREM C. For every family $\left(f_{a, \theta}\right)_{a, \theta} \in \mathcal{U}$ there exists a sequence $\left\{a_{k}\right\}$ of a-parameters accumulating in some $\bar{a}$ such that $\left(f_{a_{k}, \theta}\right)_{\theta}$ crosses the boundary of hyperbolic maps for some $\theta=\theta\left(a_{k}\right)$ strictly less than $\theta_{*}$. Moreover, $\left(f_{\bar{a}, \theta}\right)_{\theta}$ is uniformly expanding for all $\theta<\theta_{*}$.

In fact, we prove that each $f_{a_{k}, \theta}$ has a periodic hyperbolic attracting point for some $\tilde{\theta}\left(a_{k}\right)<\theta_{*}$. Since these points are robust we have an open set of parameters presenting this phenomenon inside our family. The fact that $f_{a_{k},-1}$ is uniformly expanding permits us to conclude that $f_{a_{k}, \theta}$ cross the boundary of the set of expanding maps for some $\theta\left(a_{k}\right)<\tilde{\theta}\left(a_{k}\right)<\theta_{*}$.

Our results suggest a mental picture of the shape of a piece of the border of hyperbolic maps which is reinforced by computational simulations as in Figure 1. It concerns to Arnold-like family of circle maps

$$
f_{a, \theta}(x)=k x+a+\theta \sin 2 \pi x \quad \bmod 1,
$$

with $k=2,-2 \leq a \leq 2$ and $-0.1 \leq \theta \leq 1.4$. The parameters $a$ and $\theta$ are in vertical and horizontal directions, respectively. The dark region corresponds to positive Lyapunov exponent and the light region to negative Lyapunov exponent.

1.4. Cubic critical bifurcation. Let us describe an example of an open set where our theorems hold. We say a $C^{r}$-map $\varphi: \mathbb{S}^{1} \rightarrow \mathbb{S}^{1}$ has a cubic critical point at $x=a$ if $\varphi^{\prime}(a)=0, \varphi^{\prime \prime}(a)=0$ and $\varphi^{\prime \prime \prime}(a) \neq 0$. Let $\varphi_{\theta}: \mathbb{S}^{1} \rightarrow \mathbb{S}^{1}$ be a one-parameter family of $C^{r}$-maps, $r \geq 1$. We say that $\varphi_{\theta}$ unfolds generically a cubic criticality at $(x, \theta)=$ $\left(a, \theta_{*}\right)$ if:

- $\quad \varphi_{\theta}^{\prime}(x) \neq 0$ for every $\theta<\theta_{*}$ and $x \in \mathbb{S}^{1}$;

- $\quad \varphi_{\theta_{*}}$ has a cubic critical point at $x=a$ which is its unique critical point;

- $\partial_{\theta} \varphi_{\theta}(a) \neq 0$.

The parameter $\theta$ can be thought of as the angle between the graph of $\varphi_{\theta}$ and the horizontal direction. 
Roughly, to construct an open set $\mathcal{U}$ satisfying our theorems, we construct a (central) family $\left(\tilde{f}_{\bar{a}, \theta}\right)_{\theta}$ unfolding a generic cubic criticality. The set $\mathcal{U}$ will be a $C^{r}$-open neighborhood of $\left(\tilde{f}_{a, \theta}\right)_{a, \theta}$. Observe that the existence of a cubic critical point is a robust phenomenon in this context: any family $\left(f_{a, \theta}\right)_{\theta}$ in $\mathcal{U}$ unfolds generically a cubic criticality. Let us give precise statements.

Let $f: \mathbb{S}^{1} \rightarrow \mathbb{S}^{1}$ be an orientation preserving expanding $C^{r}$-map, $r \geq 3$, that has a fixed point $p$ with a distinct pre-image $\bar{a}$.

We consider a two-parameter $C^{r}$-family $\tilde{f}_{a, \theta}: \mathbb{S}^{1} \rightarrow \mathbb{S}^{1}, \theta \in\left[\theta_{0}, \theta_{1}\right]$ and $a$ in some small open interval containing $\bar{a}$ as follows.

$\left(\mathrm{H}_{1}\right)$ For every $a$, the map $\tilde{f}_{a, \theta_{0}}$ is $C^{r}$-close to $f$ (and so is uniformly expanding).

$\left(\mathrm{H}_{2}\right)$ There is an open interval $I_{0} \subset \mathbb{S}^{1}$ containing $\bar{a}$ such that, for each $a$ and $\theta$, the maps $\tilde{f}_{a, \theta}$ and $f$ are $C^{r}$-close outside $I_{0}$.

$\left(\mathrm{H}_{3}\right)$ For every $a$, we deform $\tilde{f}_{a, \theta_{0}}$ inside an open interval $I_{a} \subset I_{0}$ centered in $a$ in such a way that the one-parameter family $\left(\tilde{f}_{a, \theta}\right)_{\theta}$ unfolds a generic cubic criticality at $\left(a, \theta_{*}\right)$.

$\left(\mathrm{H}_{4}\right)$ For all $a$ and $\theta$ we have $\partial_{a} \tilde{f}_{a, \theta}(a)>0$. Moreover, $\tilde{f}_{\bar{a}, \theta_{*}}^{\prime \prime \prime}(\bar{a}) \gg 0$ and $\tilde{f}_{a, \theta}^{\prime}(x)>1$ for every $x$ outside $I_{a}$. Furthermore we assume $f^{\prime}(p) \geq 7 / 4$.

See examples in the next section for this construction.

According to $\left(\mathrm{H}_{1}\right)$ all families $\left(\tilde{f}_{a, \theta}\right)_{\theta}$ start inside the set of uniformly expanding maps. When $\theta$ increases the family unfolds generically a cubic criticality. Observe that for each parameter $a$ the point $x=a$ is the critical point for $\tilde{f}_{a, \theta_{*}}$, and during all this process $\tilde{f}_{a, \theta}$ is expanding outside the neighborhood $I_{0}$ of $\bar{a}$; see $\left(\mathrm{H}_{2}\right)$ and $\left(\mathrm{H}_{3}\right)$. Finally, $\left(\mathrm{H}_{4}\right)$ essentially deals with a technical hypothesis and localizes the problem of loss of expansiveness inside $I_{a}$. The hypothesis on the magnitude of the derivative of $f$ in its fixed point $p$ is included just to permit explicit (numerical) bounds in some results.

The reason to make the deformation in a neighborhood of a pre-image of a fixed point of $f$ is that the lack of expansion of the derivative when $\theta$ increases to $\theta_{*}$ can be compensated with the uniform expansiveness close to the fixed point of $\tilde{f}_{a, \theta}$ where the map is uniformly expanding: the more the orbit gets close to the critical point the more it remains close to the fixed point in the next iterates. Clearly, the deformation could be done close to any higher order pre-image of the fixed point of $f$.

Our results apply to every family $\left(f_{a, \theta}\right)_{a, \theta}$ in a small $C^{r}$-neighborhood $\mathcal{U}$ of $\left(\tilde{f}_{a, \theta}\right)_{a, \theta}$. As said before, each family $\left(f_{a, \theta}\right)_{\theta}$ unfolds generically a cubic criticality, at say $(x, \theta)=$ $\left(\hat{a}, \theta_{*}\right)$. By reparametrization we can assume that $a=\hat{a}$ and take $\left[\theta_{0}, \theta_{1}\right]=[-1,1]$ and $\theta_{*}(a)=0$ for every $a$. Thus, a cubic tangency between the graph of $f_{a, \theta}$ and the horizontal direction is created on $\left(a, f_{a, \theta}(a)\right)$ at $\theta=0$.

\subsection{Examples.}

Example 1. (Figure 2) Let $f: \mathbb{S}^{1} \rightarrow \mathbb{S}^{1}$ be defined by

$$
f(x)=2 x \quad \bmod 1 .
$$

Let $\bar{a}=1 / 2$ and $p=0$. Then $\bar{a}$ is a pre-image of the fixed point $p$. Let $I_{0}=\left(x_{-}^{0}, x_{+}^{0}\right)$ be a small interval centered on $1 / 2$. Given $a$ close to $1 / 2$, let $I_{a}=\left(x_{-}^{a}, x_{+}^{a}\right)$ be a proper subinterval of $I_{0}$ centered in $a$. 


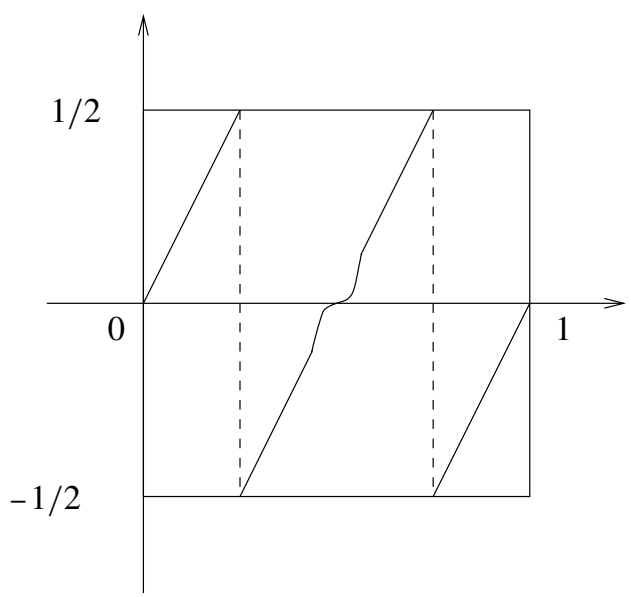

FIGURE 2. Cubic critical point.

We can define a two-parameter family $\tilde{f}_{a, \theta}, \theta \in[-1,1]$ and $a \in[-1,1]$, satisfying $\left(\mathrm{H}_{1}\right)-\left(\mathrm{H}_{4}\right)$ as follows.

(1) For every $a$, we take

$$
\tilde{f}_{a, \theta_{0}}(x)=2 x+\text { h.o.t. }(a, x) \bmod 1 .
$$

Thus, $\tilde{f}_{a, \theta_{0}}$ can be taken $C^{r}$-close to $f$.

(2) For each $a$ and $\theta$, we put for all $x \in \mathbb{S}^{1} \backslash I_{0}$

$$
\tilde{f}_{a, \theta}(x)=2 x+\text { h.o.t. }(a, \theta, x) \bmod 1 .
$$

Then, $\tilde{f}_{a, \theta}$ can be taken $C^{r}$-close to $f$ outside $I_{0}$.

(3) For every $x \in I_{a}$, we define

$$
\tilde{f}_{a, \theta}(x)=2 a-\theta(x-a)+A(x-a)^{3}+\text { h.o.t. }(a, \theta, x), \quad \bmod 1 .
$$

Then, $\tilde{f}_{a, \theta}$ unfolds a generic cubic criticality at $(0, a)$.

(4) If $\tilde{f}_{a, \theta}$ is $C^{r}$-close to $f$ outside $I_{0}$, we have $\tilde{f}_{a, \theta}^{\prime}\left(x_{ \pm}^{0}\right)>31 / 16$. On the other hand, if $\tilde{f}_{a, \theta_{*}}^{\prime \prime \prime}(\bar{a}) \approx A$ is taken large enough,

$$
\tilde{f}_{a, \theta}^{\prime}\left(x_{ \pm}^{a}\right)=-\theta+3 A\left(x_{ \pm}^{a}-a\right)^{2}>7 / 4 .
$$

Thus, it is possible to define $\tilde{f}_{a, \theta}$ in $I_{0} \backslash I_{a}$ such that $\tilde{f}_{a, \theta}^{\prime}(x) \geq \sigma>1$ for all $x \in I_{0} \backslash I_{a}$. The condition $\partial_{a} \tilde{f}_{a, \theta}(a)>0$ for every $x \in \mathbb{S}^{1}$ can easily be satisfied by these maps by taking the h.o.t. $(a)$ conveniently. Moreover, $f^{\prime}(0)=2 \geq 7 / 4$.

Next we present two examples of maps which can be used as starting points for similar constructions.

Example 2. (Arnold-like family, Figure 3) Let $k \geq 2$ be a positive integer. For $a$ small and $\theta$ close to $k /(2 \pi)$, we define

$$
\tilde{f}_{a, \theta}(x)=k x+a+\theta \sin 2 \pi x \bmod 1 .
$$




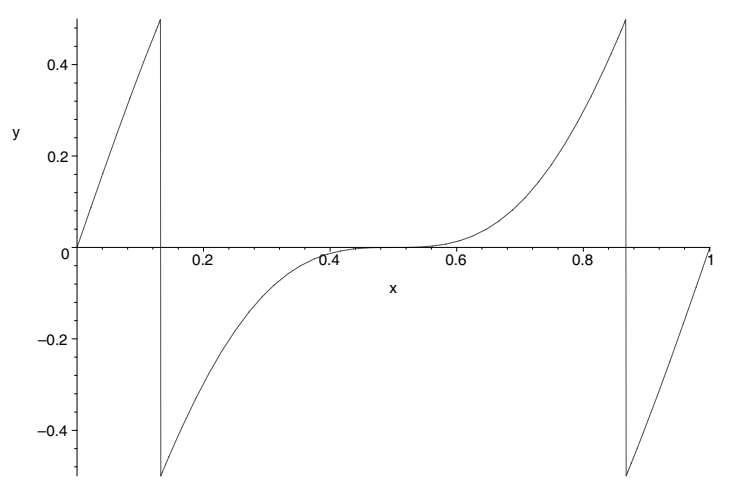

FIGURE 3. Arnold-like family, $k=2, a=0, \theta=1 / \pi$.

Let $k=2$ and $a=0$. Then, 0 is a fixed point and $1 / 2$ is a distinct pre-image of 0 for all $\theta$. Moreover, $\tilde{f}_{a, \theta}$ is uniformly expanding for $\theta=0$ and $\left(\tilde{f}_{0, \theta}\right)_{\theta}$ unfolds a cubic critical point at $\left(x, \theta_{*}\right)=(1 / 2,1 / \pi)$. By the reparametrization $a^{\prime}=a+1 / 2$ the critical point is $x=a^{\prime}$, for $\theta=1 / \pi$, satisfying $\left(\mathrm{H}_{3}\right)$. Therefore, for every $a$ sufficiently small (or after reparametrization, close to $1 / 2)\left(\tilde{f}_{a, \theta}\right)_{\theta}$ unfolds a critical cubic point at some $\left(x, \theta_{*}(a)\right)$. Up to a reparametrization of the domain of the family, we get uniform expansiveness outside a small interval centered on $1 / 2$.

Example 3. (Polynomial family, Figure 4) For a positive integer $k$ we define

$$
\tilde{f}_{a, \theta}(x)=1+2 k\left(x^{3}+a-\theta x+\theta x^{5}\right) \bmod 2
$$

where $a$ and $\theta$ belong to a neighborhood of 0 . For every $k \geq 1, x=0$ is a pre-image of the fixed point 1 and $\left(\tilde{f}_{a, \theta}\right)_{\theta}$ unfolds a critical cubic point at $(0,0)$. Indeed, this example is a $C^{\infty}$-map defined on the circle, except on a single point, and clearly does not satisfy our hypothesis. However, this family can be made smooth and satisfying the hypothesis just by a small modification near that point, and by reparametrization of the domain this family may satisfy $\left(\mathrm{H}_{1}\right)-\left(\mathrm{H}_{4}\right)$.

1.6. Ideas of the proof of Theorem A. Next, we give an idea why the proof works. We follow classical methods based on the ideas of Benedicks and Carleson $[2,3,13]$.

Note that, for $\theta<0$, the map $f_{a, \theta}(x)$ has non-zero derivative for every $x$ in $\mathbb{S}^{1}$; furthermore the unique critical point of $f_{a, 0}$ occurs for $x=a$. This motivates calling $x=a$ the critical point of $f_{a, \theta}$. We want to prove hyperbolicity (i.e. expansion) along the critical orbit, that is, the orbit of $a$.

For every $a$ and every $\theta \leq 0$ we consider the depth and frequency of returns of the critical orbit. The arguments yield a positive measure set $\Omega_{\theta}$ of $a$-parameters for which $f_{a, \theta}$ is uniformly expanding (hyperbolic) if $\theta<0$ and non-uniformly expanding if $\theta=0$. The measure of $\Omega_{\theta}$ is uniform on $\theta$ and so we get a positive Lebesgue measure subset $\mathcal{A}$ constituted by limit points of the sets $\Omega_{\theta}$ as $\theta$ goes to zero. Therefore, in this sense, the set 


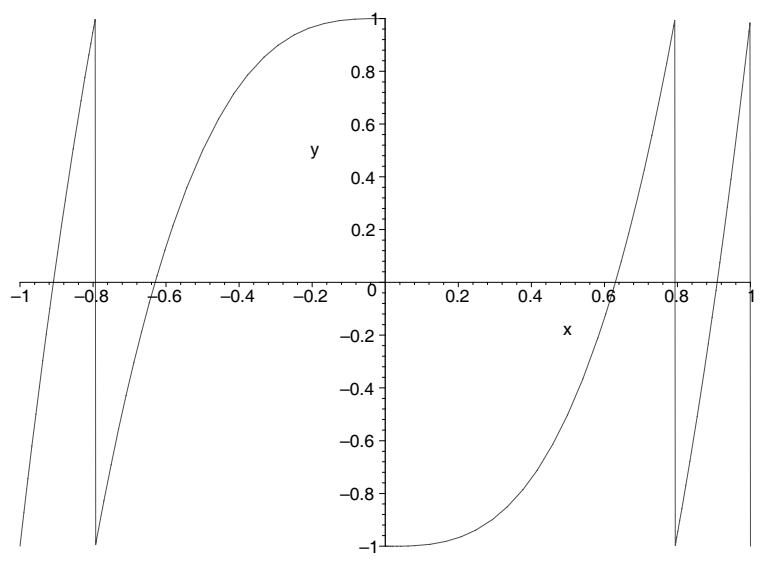

FIGURE 4. Polynomial family, $k=1, a=0, \theta=0$.

of maps $f_{a, \theta}$ such that $a$ belongs to $\mathcal{A}$ is a large subset of the boundary of the hyperbolic maps.

Now we recall the basic concepts and issues in [2] that we need to deal with. See also [15] for a careful detailed presentation of these ideas.

First of all, let $\xi_{n}(a)=f_{a, \theta}^{n}(a)$ stand for the critical orbit. We need to estimate the expansion along this orbit-later we shall see that, in this context, expansion for it implies uniform hyperbolicity of $f_{a, \theta}$.

For fixed $\delta>0$ small, we say that a positive integer $n$ is a return time for $f_{a, \theta}$ if $\left|\xi_{n}(a)-\xi_{0}(a)\right|<\delta$. In this case, as we make precise later, during a certain amount of time called a bound period the iterates $\xi_{n+1}(a), \xi_{n+2}(a), \ldots$ follow closely the critical orbit (see §3). An induction argument can be applied to transport the expansion of $f_{a, \theta}$ from $\xi_{j}(a)$ to $\xi_{n+j}(a)$ in order to recover the possible loss of expansion due to the approximation to the critical point at time $n$.

After a bound period and before the next return situation there is a period of time called a free period. The total amount of free periods before $n$ is denoted by $F_{n}(a)$.

We remark that if $\xi_{n}(a)$ becomes too close to $\xi_{0}(a)$ then a large bound period may be required and the induction can fail to work. Furthermore, the bound period is intended merely to recover the loss of expansion at time $n$ and so the final expansion is primarily obtained by the total amount $F_{n}(a)$ of free time. This gives us an idea why the two assumptions below are required for our purpose of induction.

$(\mathrm{BA})_{n}$ A recurrence control: the returns are not too close to the critical point.

$(\mathrm{FA})_{n}$ A statistical restriction: a bound of the total amount of time required to recover the loss of derivative at this time and all past times.

At each time $n$ we force the (BA) $n$ and (FA) $n$ assumptions above to be satisfied by, if necessary, excluding parameters $a$. The remaining set is denoted by $\Omega_{\theta, n}$ and the intersection of all such sets is $\Omega_{\theta}$.

Exponential growth of derivatives along the critical orbit for those parameters in $\Omega_{\theta}$ will be a consequence of the controls inductively imposed. 
2. Preliminary results

In what follows we will be working with a fixed $\theta \leq 0$ and so we will write $f_{a}(x)$ and $\xi_{n}(a, x)$ instead of $f_{a, \theta}(x)$ and $f_{a, \theta}^{n}(x)$ whenever possible. Since $f_{a}$ is $C^{1}$ and from the compactness of $\mathbb{S}^{1}$ we have $\left|\partial_{a} f_{a}\right| \leq B_{1}$ for some constant $B_{1}>0$.

LEMMA 2.1. Let

$$
Q_{n}=\frac{\partial_{a} \xi_{n}(a, x)}{\partial_{x} \xi_{n}(a, x)}
$$

Assume that:

(i) for each $x \in \mathbb{S}^{1}$ and each positive integer $n$ there exists a positive number $M_{n}(x)$ such that

$$
\left|\partial_{x} \xi_{n}(a, x)\right| \geq M_{n}(x)>0
$$

(ii) for some positive integer $\bar{N}$,

$$
2 B_{1} \sum_{n=\bar{N}}^{\infty} M_{n}^{-1} \leq\left|Q_{\bar{N}}\right|
$$

Let $Q=\max \left\{2\left|Q_{\bar{N}}\right|^{-1}, \frac{3}{2}\left|Q_{\bar{N}}\right|\right\}$. Then,

$$
Q^{-1} \leq\left|Q_{n+1}\right| \leq Q \quad \text { for every } n \geq \bar{N}
$$

Proof. By the chain rule we have

$$
\partial_{x} \xi_{n}(a, x)=\partial_{x}\left[\xi_{1}\left(a, \xi_{n-1}(a, x)\right)\right]=\partial_{x} \xi_{1}\left(a, \xi_{n-1}(a, x)\right) \partial_{x} \xi_{n-1}(a, x)
$$

and

$$
\begin{aligned}
\partial_{a} \xi_{n}(a, x) & =\partial_{a}\left[\xi_{1}\left(a, \xi_{n-1}(a, x)\right)\right] \\
& =\partial_{x} \xi_{1}\left(a, \xi_{n-1}(a, x)\right) \partial_{a} \xi_{n-1}(a, x)+\partial_{a} \xi_{1}\left(a, \xi_{n-1}(a, x)\right)
\end{aligned}
$$

Thus,

$$
Q_{n}=\frac{\partial_{a} \xi_{n}(a, x)}{\partial_{x} \xi_{n}(a, x)}=\frac{\partial_{a} \xi_{n-1}(a, x)}{\partial_{x} \xi_{n-1}(a, x)}+\frac{\partial_{a} \xi_{1}\left(a, \xi_{n-1}(a, x)\right)}{\partial_{x} \xi_{n}(a, x)}
$$

Therefore,

$$
Q_{n}-Q_{n-1}=\frac{\partial_{a} \xi_{1}\left(a, \xi_{n-1}(a, x)\right)}{\partial_{x} \xi_{n}(a, x)} .
$$

Using (i) and $\xi_{1}(a, x)=f_{a}(x)$, we can write

$$
\left|Q_{n}-Q_{n-1}\right| \leq \frac{B_{1}}{\left|\partial_{x} \xi_{n}(a, x)\right|} \leq B_{1} M_{n}^{-1}(x) .
$$

By recurrence and assumption (ii), for every $n>\bar{N}$, we have

$$
\left|Q_{n}-Q_{\bar{N}}\right| \leq B_{1} \sum_{k=\bar{N}}^{n} M_{k}^{-1}(x) \leq \frac{\left|Q_{\bar{N}}\right|}{2}
$$

Therefore,

$$
Q^{-1} \leq \frac{1}{2}\left|Q_{\bar{N}}\right|=\left|Q_{\bar{N}}\right|-\frac{\left|Q_{\bar{N}}\right|}{2} \leq\left|Q_{n+1}\right| \leq\left|Q_{\bar{N}}\right|+\frac{\left|Q_{\bar{N}}\right|}{2}=\frac{3}{2}\left|Q_{\bar{N}}\right| \leq Q .
$$


Remark 2.2. If the interval $I_{a}$ in $\left(\mathrm{H}_{3}\right)$ is taken sufficiently small, then for every $a$ close to $\bar{a}$, there exist positive constants $A_{1}<A_{2} \ll A_{3}<A_{4}$, with $A_{2}<2 A_{1}$ and $A_{4}<2 A_{3}$, such that for every $x \in I_{a}$ we have

$$
-A_{1} \theta|x-a|+A_{3}|x-a|^{3} \leq\left|f_{a, \theta}(x)-f_{a, \theta}(a)\right| \leq-A_{2} \theta|x-a|+A_{4}|x-a|^{3},
$$

where the sum is in the circle $\mathbb{S}^{1}$, and

$$
-A_{1} \theta+3 A_{3}(x-a)^{2} \leq f_{a, \theta}^{\prime}(x) \leq-A_{2} \theta+3 A_{4}(x-a)^{2} .
$$

Moreover, from $\left(\mathrm{H}_{4}\right)$, there exists $\sigma_{0}>1$ such that, for every $f_{a, \theta}$ close to $\tilde{f}_{a, \theta}$,

$$
f_{a, \theta}^{\prime}(x) \geq \sigma_{0} \quad \text { for all } x \in \mathbb{S}^{1} \backslash I_{a} .
$$

Proposition 2.3. Given $\delta>0$ sufficiently small there exist $\eta>0$, a neighborhood $\mathcal{U}$ of $\left(\tilde{f}_{a, \theta}\right)_{a, \theta}$, and a constant $c=c(\delta)>1 / 15$ such that, for every $f_{a, \theta}$ in $\mathcal{U}$, if

(a) $\left|f_{a}^{j}(x)-a\right| \geq \delta$ for $0 \leq j<k$ and

(b) $\left|f_{a}^{k}(x)-a\right|<\delta$,

then

$$
\left|\left(f_{a}^{k}\right)^{\prime}(x)\right| \geq e^{c k}
$$

whenever $|a-\bar{a}| \leq \eta$ and $-\delta^{2}<\theta \leq 0$.

Proof. Since outside $I_{a}$ the derivatives are uniformly expanding we can assume $x \in I_{a}$.

Let $p_{a}=p_{a, \theta}$ the continuation of the fixed point $p$. For some interval $J$ centered in $p_{a}$, we denote $\sigma_{m}=\inf _{x \in J}\left|f_{a}^{\prime}(x)\right|$ and $\sigma_{M}=\sup _{x \in J}\left|f_{a}^{\prime}(x)\right|$. We fix $J$ such that $|J|<1 / 4$ and

$$
l_{0}=\frac{\log \sigma_{m}}{\log \sigma_{M}}>\frac{8}{9} .
$$

By choosing $\eta$ small we get $f_{a}\left(I_{a}\right) \subset J$ for all $a$ in $(\bar{a}-\eta, \bar{a}+\eta)$.

Since $f_{a}(x)$ is close to $p_{a}$, during a certain amount of time the orbit of $x$ remains in $J$. We can suppose $f_{a}(x) \neq p_{a}$, otherwise the result is trivial. If $k_{0}$ is the first iterate of $x$ that escapes from $J$ we must have

$$
\sigma_{M}^{k_{0}}\left|f_{a}(x)-p_{a}\right| \geq|J| \text { and } \sigma_{M}^{k_{0}-1}\left|f_{a}(x)-p_{a}\right| \leq|J| .
$$

At this moment we have

$$
\left|\left(f_{a}^{k_{0}+1}\right)^{\prime}(x)\right|=\left|\left(f_{a}^{k_{0}}\right)^{\prime}\left(f_{a}(x)\right)\right|\left|f_{a}^{\prime}(x)\right| \geq \sigma_{m}^{k_{0}}\left|f_{a}^{\prime}(x)\right| .
$$

In order to estimate the term $\sigma_{m}^{k_{0}}$ we observe from (4) that

$$
k_{0} \geq \frac{\log \left(|J| /\left|f_{a}(x)-p_{a}\right|\right)}{\log \sigma_{M}}
$$

and so

$$
\log \sigma_{m}^{k_{0}} \geq \frac{\log \left(|J| /\left|f_{a}(x)-p_{a}\right|\right)}{\log \sigma_{M}} \log \sigma_{m}=\log \left(|J| /\left|f_{a}(x)-p_{a}\right|\right)^{l_{0}} .
$$

The derivative on the right-hand side of (5) can be estimated, using (2), as

$$
\left|f_{a}^{\prime}(x)\right| \geq-A_{1} \theta+3 A_{3}|x-a|^{2} \geq 3 A_{3}|x-a|^{2} .
$$


Therefore, replacing (6) and (7) in (5), we have

$$
\left|\left(f_{a}^{k_{0}+1}\right)^{\prime}(x)\right| \geq|J|^{l_{0}} \frac{3 A_{3}|x-a|^{2}}{\left|f_{a}(x)-p_{a}\right|^{l_{0}}} .
$$

First, we estimate

$$
\left|f_{a}(x)-p_{a}\right| \leq\left|f_{a}(x)-f_{a}(a)\right|+\left|f_{a}(a)-p_{a}\right| .
$$

Since $f_{a}$ is close to $\tilde{f}_{a}$ and taking $\eta$ sufficiently small, then (recall that $\tilde{f}_{\bar{a}}(\bar{a})=p$ ) we have

$$
\left|f_{a}(a)-p_{a}\right| \leq\left|f_{a}(a)-\tilde{f}_{a}(a)\right|+\left|\tilde{f}_{a}(a)-\tilde{f}_{a}(\bar{a})\right|+\left|\tilde{f}_{a}(\bar{a})-\tilde{f}_{\bar{a}}(\bar{a})\right|+\left|p-p_{a}\right| \leq \delta^{3} .
$$

From (1), (9) and (10) and assuming $|x-a|>\delta$ and $\delta^{2}>-\theta$, we get

$$
\begin{aligned}
\left|f_{a}(x)-p_{a}\right| & \leq-A_{2} \theta|x-a|+A_{4}|x-a|^{3}+\delta^{3} \\
& \leq\left(A_{2}+A_{4}+1\right)|x-a|^{3} \leq 3 A_{4}|x-a|^{3}<6 A_{3}|x-a|^{3} .
\end{aligned}
$$

From this latter estimate, (8) and (4), we get

$$
\begin{aligned}
\left|\left(f_{a}^{k_{0}+1}\right)^{\prime}(x)\right| & \geq \frac{|J|^{l_{0}} 3 A_{3}|x-a|^{2}}{\left|f_{a}(x)-p_{a}\right|^{2 / 3}\left|f_{a}(x)-p_{a}\right|^{l_{0}-2 / 3}} \geq \frac{|J|^{l_{0}} 3 A_{3}}{\left(6 A_{3}\right)^{2 / 3}\left|f_{a}(x)-p_{a}\right|^{l_{0}-2 / 3}} \\
& \geq \frac{1}{2}|J|^{8 / 9}\left(6 A_{3}\right)^{1 / 3} \sigma_{M}^{\left(k_{0}-1\right)\left(l_{0}-2 / 3\right)} .
\end{aligned}
$$

Since we can suppose $A_{3} \gg 1$ (independent of $|J|$ ), then we get

$$
\left|\left(f_{a}^{k_{0}+1}\right)^{\prime}(x)\right| \geq \sigma_{M}^{\left(k_{0}-1\right)\left(l_{0}-2 / 3\right)}=e^{c\left(k_{0}+1\right)},
$$

where

$$
c=\frac{k_{0}-1}{k_{0}+1}\left(l_{0}-\frac{2}{3}\right) \log \sigma_{M}>\frac{1}{6} \log \sigma_{M}>\frac{1}{15} .
$$

The last two inequalities use $k_{0} \geq 7$ and $\sigma_{M} \geq 3 / 2$; recall by $\left(\mathrm{H}_{4}\right)$ we are assuming $f^{\prime}(p) \geq 7 / 4$.

Note that we can choose the neighborhood $J$ of $p$ in such a way that $f^{k_{0}+1}(x) \notin I_{a}$. To finish the proof we observe that for all $0 \leq j<k$ such that $f_{a}^{j}(x)$ belongs to $I_{a}$ we can use the same arguments as before since due to hypothesis (b) we know that $j+k_{0}<k$. This completes the proof of Proposition 2.3.

Remark 2.4. Without assuming (b) in the preceding lemma we can easily obtain an estimate for the expanding behavior of $f_{a}$ like

$$
\left|\left(f_{a}^{k}\right)^{\prime}(x)\right| \geq \delta^{2} e^{c k}
$$

Remark 2.5. The inductive method we use to prove Theorem A uses repeatedly an idea present in the preceding lemma: orbits $\left\{f_{a}^{n}(a)\right\}_{n}$ spend an arbitrarily large amount $N$ of time near the fixed point of $f_{a}$ provided we consider parameters $a$ very close to $\bar{a}$. 


\section{The bound period}

Let $(a, \theta)$ be fixed and consider the orbits of $f_{a, \theta}$. Whenever an iterate of $x$ gets close to $a$, we have some loss on the expansion of the derivative. On the other hand, by continuity, during some few iterates the orbits of $x$ and $a$ will remain close. Taking into account this basic fact and assuming as an inductive hypothesis that the orbit of $a$ is expanding (at least during these iterates) we may end up with the trajectory of $x$ recovering its eventual loss on the expansion of its derivative.

In order to present more details about this approach, we are going to introduce some terminology.

Fix $\delta=e^{-(\Delta+1)}$, for some $\Delta \in \mathbb{N}$, in such a way that all the results in the previous sections concerning $\delta$ are satisfied.

Given a trajectory $f_{a, \theta}^{n}(x)$, we can split it using the following rough approach.

- $\quad$ Return times. Those iterates when $f_{a, \theta}^{n}(x)$ gets close to $a$. More precisely, we state that $n$ is a return time for the orbit of $x$ if we have $\left|f_{a, \theta}^{n}(x)-a\right|<\delta$.

- $\quad$ Bound period. The piece of orbit of $x$ that remains close to the orbit of $a$. This is accomplished by fixing $\beta$ small and defining

$$
p(x, a)=\max \left\{k:\left|f_{a, \theta}^{n+j}(x)-f_{a, \theta}^{j}(a)\right| \leq e^{-\beta j}, 0 \leq j \leq k\right\} .
$$

The bound period associated to the return $n$ of $x$ is the set of times $n+1, \ldots, n+p$, $p=p(x, a)$.

- $\quad$ Free period. Those iterates after a return and its corresponding bound period and before the next return time.

We point out that it is possible to have $\left|f_{a, \theta}^{n}(x)-a\right|<\delta$ for iterates $n$ classified above as part of a bound period of a previous return. We say that $n$ corresponds to a bound return and they can be securely ignored as a consequence of the estimates for the bound period present in Proposition 3.1.

Note that we have presented these notions for a single orbit. On the other hand, we will need to consider groups of orbits which behave similarly during a certain amount of time and this yields the idea of partitioning the phase space around a critical point in order to achieve this control. We introduce now a model of this type of partitioning which will be slightly reviewed when we consider joint dynamics of an interval of parameters, in the next section.

Let $a$ be a parameter under consideration. We can think of $a$ also as a point in the phase space and define a partition of a neighborhood of $a$ by disjoint intervals $I_{m}$ given by

$$
I_{m}=\left[a+e^{-(m+1)}, a+e^{-m}\right), \quad m \geq \Delta .
$$

We extend this definition to negative indices by imposing that $I_{-m}$ and $I_{m}$ be symmetrical with respect to $a$. Additionally we write

$$
U_{m}=\left(a-e^{-m}, a+e^{-m}\right), \quad m \geq \Delta .
$$

For this partition we write (whenever it makes sense)

$$
U_{m}^{+}(a)=I_{-(m-1)} \cup U_{m} \cup I_{m-1} \quad \text { and } \quad I_{m}^{+}(a)=I_{m-1} \cup I_{m} \cup I_{m+1} .
$$


As a refinement of this procedure, we divide each interval $I_{m}$ into $m^{2}$ subintervals of equal length which will be denoted by $I_{m, k}, k=1, \ldots, m^{2}$. After all this we have

$$
U_{\Delta}(a)=\left\{\bigcup I_{m, k}: m \geq \Delta, 1 \leq k \leq m^{2}\right\} \cup\{a\} .
$$

For technical issues we join to that collection of intervals these two additional ones:

$$
I_{\Delta-1,1}=\left[a+e^{-\Delta}, a+e^{-\Delta}+\tau\right) \quad \text { and } \quad I_{-(\Delta-1), 1}=\left(a-e^{-\Delta}-\tau, a-e^{-\Delta}\right],
$$

where $\tau=\left|e^{-(\Delta-1)}-e^{-\Delta}\right| /(\Delta-1)^{2}$.

Again, taking into account this refinement, we reserve the plus sign (+) to indicate, e.g., by $I_{m, k}^{+}$, the union of an interval with its two adjacent ones.

At this point let us extend the previous definition of bound period by writing

$$
p(m, a)=\min _{x \in U_{m}^{+}} p(x, a) .
$$

Note that for some points in $U_{m}^{+}$we can end up with a shorter bound period than before. Since we need to recover completely the loss of derivatives at return times, we would like to state that this modification does not affect the essential properties of the bound period (see the next proposition).

At this point we need to make precise the notions of recurrence control and exponential growth introduced in $\S 1.6$. We fix $\alpha<\beta$, say $\alpha=\beta / 10$, and for $j \in \mathbb{N}$ we state these assumptions as

$$
\begin{aligned}
& (\mathrm{BA})_{j}:\left|\xi_{j}(a)-a\right| \geq e^{-\alpha j}, \\
& (\mathrm{EG})_{j}:\left|\left(f_{a, \theta}^{j}\right)^{\prime}(a)\right| \geq e^{c j},
\end{aligned}
$$

where $c$, the rate of exponential growth, can be taken essentially equal to that given by Proposition 2.3.

Proposition 3.1. Let a be a parameter which satisfies $(B A)_{j}$ and $(E G)_{j}$ for $j=$ $1, \ldots, n$, with $n \geq 1$. Let $\Delta \leq|m| \leq[\alpha n]$ and write $p=p(m, a)$. Then, there is $a$ constant $C_{1}=C_{1}(\alpha)$ such that:

(a) $\frac{1}{C_{1}} \leq \frac{\left|\left(f_{a, \theta}^{j}\right)^{\prime}\left(f_{a, \theta}(x)\right)\right|}{\left|\left(f_{a, \theta}^{j}\right)^{\prime}\left(f_{a, \theta}(a)\right)\right|} \leq C_{1} \quad$ for $j=1, \ldots, p$ and every $x \in U_{m}^{+}$;

(b) $\quad p<50|m| \ll n$;

(c) $\left|\left(f_{a, \theta}^{p+1}\right)^{\prime}(x)\right| \geq e^{(1-50 \beta)|m|}$ for every $x \in I_{m}^{+}$.

Proof. (a) Let $x_{i}=f_{a, \theta}^{i}(x)$ and $a_{i}=f_{a, \theta}^{i}(a)$. Given $j=1, \ldots, \min (p, n)$ we have

$$
\frac{\left|\left(f_{a, \theta}^{j}\right)^{\prime}\left(f_{a, \theta}(x)\right)\right|}{\left|\left(f_{a, \theta}^{j}\right)^{\prime}\left(f_{a, \theta}(a)\right)\right|}=\prod_{i=1}^{j} \frac{\left|f_{a, \theta}^{\prime}\left(f_{a, \theta}^{i}(x)\right)\right|}{\left|f_{a, \theta}^{\prime}\left(f_{a, \theta}^{i}(a)\right)\right|}=\prod_{i=1}^{j}\left|1+\frac{f_{a, \theta}^{\prime}\left(x_{i}\right)-f_{a, \theta}^{\prime}\left(a_{i}\right)}{f_{a, \theta}^{\prime}\left(a_{i}\right)}\right| .
$$

Case 1. If $a_{i} \notin I_{a}$ then we have $f_{a, \theta}^{\prime}\left(a_{i}\right) \geq \sigma_{0}>1$, recall (3), and since $i \leq j \leq p$, we get, for some constant $K>0$,

$$
\left|\frac{f_{a, \theta}^{\prime}\left(x_{i}\right)-f_{a, \theta}^{\prime}\left(a_{i}\right)}{f_{a, \theta}^{\prime}\left(a_{i}\right)}\right| \leq K e^{-\beta i}
$$


Case 2. If $a_{i} \in I_{a}$ then we get, for some positive constant $K^{\prime}$,

$$
\left|f_{a, \theta}^{\prime}\left(x_{i}\right)-f_{a, \theta}^{\prime}\left(a_{i}\right)\right| \leq K^{\prime}\left|x_{i}-a_{i}\right| .
$$

Since $i \leq n$ we have that $a$ satisfies (BA) ${ }_{i}$ for all such $i$. From $i \leq p$ and from Remark 2.2, we get

$$
\left|\frac{f_{a, \theta}^{\prime}\left(x_{i}\right)-f_{a, \theta}^{\prime}\left(a_{i}\right)}{f_{a, \theta}^{\prime}\left(a_{i}\right)}\right| \leq \frac{K^{\prime}\left|x_{i}-a_{i}\right|}{-A_{1} \theta+3 A_{3}\left(a_{i}-a\right)^{2}} \leq \frac{K^{\prime} e^{-\beta i}}{e^{-2 \alpha i}} \leq K^{\prime} e^{-(\beta-2 \alpha) i} .
$$

Note that for $i$ sufficiently large the term above is less than 1 ( $\beta=10 \alpha)$. Hence, from (12), we have

$$
\begin{aligned}
\prod_{i=1}^{j}\left(1-\left|\frac{f_{a, \theta}^{\prime}\left(x_{i}\right)-f_{a, \theta}^{\prime}\left(a_{i}\right)}{f_{a, \theta}^{\prime}\left(a_{i}\right)}\right|\right) & \leq \frac{\left|\left(f_{a, \theta}^{j}\right)^{\prime}\left(f_{a, \theta}(x)\right)\right|}{\left|\left(f_{a, \theta}^{j}\right)^{\prime}\left(f_{a, \theta}(a)\right)\right|} \\
& \leq \prod_{i=1}^{j}\left(1+\left|\frac{f_{a, \theta}^{\prime}\left(x_{i}\right)-f_{a, \theta}^{\prime}\left(a_{i}\right)}{f_{a, \theta}^{\prime}\left(a_{i}\right)}\right|\right) .
\end{aligned}
$$

Therefore from (13) and (14) it follows that there is a constant $C_{1}>0$ such that

$$
\frac{1}{C_{1}} \leq \frac{\left|\left(f_{a, \theta}^{j}\right)^{\prime}\left(f_{a, \theta}(x)\right)\right|}{\left|\left(f_{a, \theta}^{j}\right)^{\prime}\left(f_{a, \theta}(a)\right)\right|} \leq C_{1} .
$$

In order to conclude the proof of part (a), we have to verify that $p<n$. This is a consequence of the next item.

(b) For simplicity of notation let us suppose $m>0$ (the other case is analogous). Let $x_{m}=a+e^{-m}$ be in the boundary of $I_{m} \subset\left(U_{m}^{+}\right)$and let $j=\min \{n, p\}-1$. Then, there exists $y \in\left(f_{a, \theta}(a), f_{a, \theta}\left(x_{m}\right)\right)$ such that

$$
\left|f_{a, \theta}\left(x_{m}\right)-f_{a, \theta}(a)\right|\left|\left(f_{a, \theta}^{j}\right)^{\prime}(y)\right|=\left|f_{a, \theta}^{j+1}\left(x_{m}\right)-f_{a, \theta}^{j+1}(a)\right|<e^{-\beta(j+1)} .
$$

From the fact that $a$ satisfies (EG) $j$ we get

$$
\left|\left(f_{a, \theta}^{j}\right)^{\prime}(a)\right| \geq e^{c j}
$$

and so, by part (a), we can conclude that

$$
\left|\left(f_{a, \theta}^{j}\right)^{\prime}(y)\right| \geq C_{1}^{-1} e^{c j} .
$$

On the other hand, from the local form (1), we have

$$
\begin{aligned}
\left|f_{a, \theta}\left(x_{m}\right)-f_{a, \theta}(a)\right| & \geq-A_{1} \theta\left|x_{m}-a\right|+A_{3}\left|x_{m}-a\right|^{3} \\
& \geq A_{3}\left|x_{m}-a\right|^{3} \geq A_{3} e^{-3 m} .
\end{aligned}
$$

From (15) and (16) we have

$$
C_{1}^{-1} e^{c j} A_{3} e^{-3 m} \leq e^{-\beta(j+1)} .
$$

Then,

$$
\log \left(C_{1}^{-1} A_{3}\right)-3 m+c j \leq-\beta(j+1)
$$


Thus, as $C_{1}^{-1} A_{3}>1\left(A_{3} \gg 0\right)$, we have (recall that $\left.c>1 / 15\right)$

$$
j+1<\frac{3 m-\beta}{c+\beta}+1<50 m \leq 50 \alpha n \ll n .
$$

In particular $j=p-1$ and so

$$
p \leq 50 m
$$

as required.

(c) Again, without loss of generality, we suppose $m>0$. It follows from the mean value theorem that there exists $y \in U_{m}^{+}$such that

$$
\left|\left(f_{a, \theta}^{p+1}\right)\left(U_{m}^{+}\right)\right|=\left|\left(f_{a, \theta}^{p+1}\right)^{\prime}(y)\right|\left|U_{m}^{+}\right|=\left|\left(f_{a, \theta}^{p}\right)^{\prime}\left(f_{a, \theta}(y)\right)\right|\left|f_{a, \theta}^{\prime}(y)\right|\left|U_{m}^{+}\right| .
$$

Let $x$ be an arbitrary point of $I_{m}^{+}$. Then, by part (a),

$$
\left|\left(f_{a, \theta}^{p+1}\right)^{\prime}(x)\right|=\left|\left(f_{a, \theta}^{p}\right)^{\prime}\left(f_{a, \theta}(x)\right)\right|\left|f_{a, \theta}^{\prime}(x)\right| \geq \frac{1}{C_{1}}\left|\left(f_{a, \theta}^{p}\right)^{\prime}\left(f_{a, \theta}(y)\right)\right|\left|f_{a, \theta}^{\prime}(x)\right| .
$$

The fact that $\left|f_{a, \theta}^{p+1}\left(U_{m}^{+}\right)\right| \geq e^{-\beta(p+1)}$ and $\left|U_{m}^{+}\right|<2 e^{-m+1}$ implies that

$$
\left|\left(f_{a, \theta}^{p+1}\right)^{\prime}(x)\right| \geq \frac{1}{C_{1}} \frac{\left|f_{a, \theta}^{p+1}\left(U_{m}^{+}\right)\right|}{\left|U_{m}^{+}\right|} \frac{\left|f_{a, \theta}^{\prime}(x)\right|}{\left|f_{a, \theta}^{\prime}(y)\right|} \geq \frac{1}{2 C_{1}} \frac{e^{-\beta(p+1)}}{e^{-m+1}} \frac{\left|f_{a, \theta}^{\prime}(x)\right|}{\left|f_{a, \theta}^{\prime}(y)\right|} .
$$

From the local form (2), we have

$$
\frac{f_{a, \theta}^{\prime}(x)}{f_{a, \theta}^{\prime}(y)} \geq \frac{-A_{1} \theta+3 A_{3}(x-a)^{2}}{-A_{2} \theta+3 A_{4}(y-a)^{2}} \geq \frac{-A_{1} \theta+3 A_{3} e^{-2 m}}{-A_{2} \theta+3 A_{4} e^{-2 m+2}} .
$$

For $\theta=0$, we have

$$
\frac{f_{a, 0}^{\prime}(x)}{f_{a, 0}^{\prime}(y)} \geq \frac{A_{3}}{A_{4}} e^{-2} .
$$

By continuity of $f_{a, \theta}^{\prime}$ and by reducing $\theta_{0}$ if necessary, we have, for every negative $\theta$ close to 0 ,

$$
\frac{f_{a, \theta}^{\prime}(x)}{f_{a, \theta}^{\prime}(y)} \geq \frac{A_{3}}{A_{4}} e^{-3} .
$$

Finally, it follows from (18), (19) and part (b) that

$$
\left|\left(f_{a, \theta}^{p+1}\right)^{\prime}(x)\right| \geq \frac{A_{3} e^{-3}}{2 A_{4} C_{1}} \frac{e^{-\beta(p+1)}}{e^{-m+1}} \geq e^{(1-50 \beta) m} .
$$

\section{The induction process}

Since we follow classical techniques we just outline the proof when it is analogous to the quadratic case. Emphasis is put on those steps where we deal with the specific cubic setting.

In all that follows, we consider $\theta \leq 0$ fixed and so let us write $f_{a}=f_{a, \theta}$.

Recall that we have an initial interval of parameters $\Omega_{0}=\Omega_{\theta, 0}=[\bar{a}-\eta, \bar{a}+\eta]$ (where $\eta$ satisfies Propositions 2.3 and 3.1) from which we want to extract a positive 
Lebesgue measure subset of parameters satisfying the conclusions of Theorem A. In order to obtain such a subset we make some inductive parameter exclusions. For this reason we have to consider the dynamics of an interval of parameters instead of a single parameter. Thus, we have to get similar results of previous sections for whole intervals of parameters.

We construct a sequence of partitions $\left(\mathcal{P}_{n}\right)_{n}$, where each partition $\mathcal{P}_{n}=\mathcal{P}_{\theta, n}$ is a collection of intervals $\omega$. First of all, we put $\mathcal{P}_{0}=\{\omega\}$, with $\omega=\Omega_{0}$. Now suppose that $\mathcal{P}_{0}, \mathcal{P}_{1}, \ldots, \mathcal{P}_{n-1}$ are defined and let us describe how to refine $\mathcal{P}_{n-1}$ in order to construct $\mathcal{P}_{n}$. For future references we write

$$
\Omega_{k}=\Omega_{\theta, k}=\bigcup_{\omega \in \mathcal{P}_{k}} \omega .
$$

Given $\omega=\left[\omega_{-}, \omega_{+}\right] \in \mathcal{P}_{n-1}$, we define a partition of the neighborhood of radius $\delta$ of $\omega$ in exactly the same way as we did before for a single parameter $a$ (see $\S 3$, especially (11)). For example, we write,

$$
U_{m}(\omega)=\left(\omega_{-}-e^{-m}, \omega_{+}+e^{-m}\right) \quad \text { and } \quad I_{m}(\omega)=\left[\omega_{+}+e^{-(m+1)}, \omega_{+}+e^{-m}\right) .
$$

Following the referred section the meanings of $I_{m, k}(\omega), I_{m, k}^{+}(\omega)$ and so on should be clear. From now on, we omit $\omega$ from the notation whenever the context permits.

In order to describe the construction of $\mathcal{P}_{n}$ we have to discuss the fate of such an interval $\omega$ in $\mathcal{P}_{n-1}$ when we pass from time $n-1$ to time $n$. The dynamics of $\omega$ will be given by

$$
\xi(\omega)=\bigcup_{a \in \omega}\left\{f_{a}(a)\right\}
$$

and, more generally,

$$
\xi_{j}(\omega)=\bigcup_{a \in \omega}\left\{f_{a}^{j}(a)\right\} .
$$

At time $n$, if $\xi_{n}(\omega)$ does not intersect $U_{\Delta}$ we put $\omega$ in $\mathcal{P}_{n}$; otherwise we distinguish two cases. In the first one, if $\xi_{n}(\omega)$ does not cover completely any $I_{m, k}$ we say that $n$ is an inessential return time for $\omega$ with host interval $I_{\bar{m}, \bar{k}}$ where $\xi_{n}(\omega) \subset I_{\bar{m}, \bar{k}}^{+}$. In the remaining case, we say that $n$ is an essential return time for $\omega$ and we have a natural way of inducing a partition of it by considering those subintervals of $\omega$ whose iterates are intervals $I_{m, k}$ defined earlier. We write

$$
\omega=\bigcup \omega_{m, k}^{\prime},
$$

where each $\omega_{m, k}^{\prime}$ is an interval mapped by $\xi_{n}$ into $I_{m, k}$. In the case where the extremal intervals of $\omega$ do not cover completely an interval $I_{m, k}$ we join them to its adjacent ones. After all this, each $\omega_{m, k}^{\prime}$ is put in $\mathcal{P}_{n}$ and assigned to the host interval $I_{m, k}$.

Finally, each subinterval $\omega^{\prime}$ of $\omega$ in the case of an essential return and $\omega$ itself in the case of an inessential one will be included in $\mathcal{P}_{n}$ if, and only if, it satisfies a recurrence control and a statistical restriction to be explained in a little while.

We have already defined the concept of return times for intervals $\omega$. The notion of bound period for $\omega$ with a host interval $I_{m, k}$ is defined by

$$
p(m, \omega)=\min _{a \in \omega} p(m, a) .
$$

The free periods are the complement of return times and bound periods. 
In order to avoid overload of notation let us keep denoting (BA) $n$ the recurrence control and $(\mathrm{FA})_{n}$ the statistical restriction for an interval of parameters $\omega$. Let us make these concepts precise:

$(\mathrm{BA})_{n}: \xi_{n}(\omega) \cap U_{[\alpha n]}(\omega)=\emptyset$,

$(\mathrm{FA})_{n}: F_{a}(n) \geq(1-\alpha) n$, for all $a \in \omega$,

where $F_{a}(n)$ stands for the amount of free iterates up to time $n$. More precisely, each $\omega_{n} \in \mathcal{P}_{n}$ has a sequence of ancestors,

$$
\omega_{1} \supset \omega_{2} \supset \cdots \supset \omega_{n},
$$

with $\omega_{i} \in \mathcal{P}_{i}$ and the concept of free iterates refers to the dynamics of these intervals.

Additionally, let us write

$$
(\mathrm{EG})_{n}:\left|\left(f_{a}^{j}\right)^{\prime}(a)\right| \geq e^{c j} \text {, for } 1 \leq j \leq n \text { and for all } a \in \omega \text {. }
$$

Once we have established that (21) is in fact an appropriate definition of bound period for all parameters $a$ in $\omega \in \mathcal{P}_{n-1}$ (that is to say, the content of Proposition 3.1 remains valid when we think of $\omega$ instead of a single parameter $a$ ) then we can use the methods of Benedicks and Carleson in $[2,3,13]$. We suppose at time $n-1$ that all $\omega$ in $\mathcal{P}_{n-1}$ satisfy $(\mathrm{BA})_{n-1},(\mathrm{FA})_{n-1}$ and $(\mathrm{EG})_{n-1}$ then choose for $\mathcal{P}_{n}$ only the subintervals $\omega^{\prime}$ of $\omega$ as in (20) satisfying $(\mathrm{BA})_{n}$ and $(\mathrm{FA})_{n}$. From that argument, we have

$$
(\mathrm{BA})_{n}+(\mathrm{FA})_{n} \Longrightarrow(\mathrm{EG})_{n}
$$

Remark 4.1. It is important to stress now that the classical arguments guarantee that the exponential growth of the derivative until the time $n-1$ supposed by inductive hypothesis can be extended to the time $n$ by imposing the recurrence control and the statistical restriction. Hence, after pass $n$ of our induction, whenever we refer to $\omega \in \mathcal{P}_{k}$, with $k \leq n$, this implies $\left|\left(f_{a}^{j}\right)^{\prime}(a)\right| \geq e^{c j}$ for all $j$ up to time $k$ and for all $a \in \omega$.

This strategy yields a parameter set

$$
\Omega_{\theta}=\bigcap_{n \in \mathbb{N}} \Omega_{n},
$$

whose associated maps are all expanding along its critical orbit. Moreover, after we have estimated the total measure of parameter intervals excluded we will be able to conclude that $\operatorname{Leb}\left(\Omega_{\theta}\right)>0$.

Now we have to prove an analog of Proposition 3.1 for $p(\omega, m)$ as given in (21). A simple analysis shows us that items (a) and (b) remain valid but (c) must be reestablished. The trouble in this context refers to steps of the proof that deal with estimates for $\left|U_{m}^{+}\right|$ (see (18)). Note that, in this new setting,

$$
\left|U_{m}^{+}\right|=2 e^{-m}+|\omega| .
$$

Thus, it is necessary that $|\omega|$ be very small with respect to $e^{-m}$ in order that we can use the same arguments as before. That will be a consequence of the inductive construction (which enforces $(\mathrm{BA})_{n}$ and the next lemma).

LEMMA 4.2. If $\omega \in \mathcal{P}_{n}$ then $|\omega| \leq C_{3} e^{-c n}$ for some constant $C_{3}>0$ and all $n$ sufficiently large. 
Proof. We have, for some $a \in \omega$,

$$
\operatorname{deg}(f) \geq\left|\xi_{n}(\omega)\right|=\left|\xi_{n}^{\prime}(a)\right||\omega|
$$

Since we are taking $a$ in $\omega \in \mathcal{P}_{n}$, we know, from our inductive construction, that $\left|\left(f_{a}^{j}\right)^{\prime}(a)\right| \geq e^{c j}$, for $1 \leq j \leq n$. This enables us to use Lemma 2.1 to get

$$
\left|\xi_{n}^{\prime}(a)\right| \geq Q^{-1}\left|\left(f_{a}^{n}\right)^{\prime}(a)\right| \geq Q^{-1} e^{c n}
$$

and so

$$
|\omega| \leq \operatorname{deg}(f) Q e^{-c n} .
$$

From the previous lemma and (22) we can state for all $\omega \in \mathcal{P}_{n}, n$ sufficiently large, that

$$
\left|U_{m}^{+}\right| \leq 4 e^{-m}
$$

and so the arguments in the proof of Proposition 3.1 which rely on the size of $U_{m}^{+}$can be reproduced in this new context.

In order to state that item (c) holds in this new setting we begin by noting that $p(\omega, m)=p(a, m)$ for some $a \in \omega$. Therefore, writing $p=p(\omega, m)$, we have

$$
\left|\left(f_{a}^{p+1}\right)^{\prime}(x)\right| \geq e^{(1-50 \beta)|m|} .
$$

We have to prove that a similar estimate still holds with another arbitrary parameter $b$ in place of $a$. To accomplish this, we need to prove that derivatives during the bound period are comparable in space parameter.

Let $b \in \omega$ be arbitrary. Then

$$
\frac{\left(f_{a}^{p+1}\right)^{\prime}(x)}{\left(f_{b}^{p+1}\right)^{\prime}(y)}=\prod_{j=1}^{p+1} \frac{f_{a}^{\prime}\left(f_{a}^{j}(x)\right)}{f_{b}^{\prime}\left(f_{b}^{j}(y)\right)}=\prod_{j=1}^{p+1}\left(1+\frac{f_{a}^{\prime}\left(f_{a}^{j}(x)\right)-f_{b}^{\prime}\left(f_{b}^{j}(y)\right)}{f_{b}^{\prime}\left(f_{b}^{j}(y)\right)}\right) .
$$

We write $x_{a, j}=f_{a}^{j}(a)$ and $y_{b, j}=f_{b}^{j}(b)$. Taking into account that the maps $f_{a}$ and $f_{b}$ are $C^{2}$ we can choose $z_{j}$ between $x_{a, j}$ and $y_{b, j}, t \in[a, b] \subset \omega$ and a positive constant $K$ such that

$$
\begin{aligned}
\frac{\left|f_{a}^{\prime}\left(x_{a, j}\right)-f_{b}^{\prime}\left(y_{b, j}\right)\right|}{\left|f_{b}^{\prime}\left(y_{b, j}\right)\right|} & =\frac{\left|f_{a}^{\prime}\left(x_{a, j}\right)-f_{a}^{\prime}\left(y_{b, j}\right)+f_{a}^{\prime}\left(y_{b, j}\right)-f_{b}^{\prime}\left(y_{b, j}\right)\right|}{\left|f_{b}^{\prime}\left(y_{b, j}\right)\right|} \\
& \leq \frac{\left|f_{a}^{\prime \prime}\left(z_{j}\right)\right|\left|x_{a, j}-y_{b, j}\right|+\left|\partial_{a} f_{t}^{\prime}\left(y_{b, j}\right)\right||a-b|}{\left|f_{b}^{\prime}\left(y_{b, j}\right)\right|} \\
& \leq \frac{K\left(\left|x_{a, j}-y_{b, j}\right|+|a-b|\right)}{\left|f_{b}^{\prime}\left(y_{b, j}\right)\right|} .
\end{aligned}
$$

We have $\left|f_{b}^{\prime}\left(y_{b, j}\right)\right| \geq 3 A_{3}\left|y_{b, j}-b\right|^{2}$ and

$$
\begin{aligned}
\left|y_{b, j}-b\right| & \geq\left|f_{b}^{j}(b)-b+y_{b, j}-f_{b}^{j}(b)\right| \\
& \geq e^{-\alpha j}-e^{-\beta j} \geq\left(1-e^{-(\beta-\alpha) j}\right) e^{-\alpha j} .
\end{aligned}
$$


Moreover, from our definition of bound period, and since $x, y \in I_{m}^{+}$and $a, b \in \omega$, we have

$$
\left|x_{a, j}-y_{b, j}\right|+|a-b| \leq \tilde{C}\left(e^{-\beta j}+e^{-c n}\right)<2 \tilde{C} e^{-\beta j}, \quad j=1, \ldots, p,
$$

for some $\tilde{C}>0$.

From these last two estimates we have

$$
\frac{\left|f_{a}^{\prime}\left(x_{a, j}\right)-f_{b}^{\prime}\left(y_{b, j}\right)\right|}{\left|f_{b}^{\prime}\left(y_{b, j}\right)\right|} \leq 4 K\left(3 A_{3}\right)^{-1} \tilde{C} e^{-(\beta-2 \alpha) j}
$$

and so

$$
\sum_{j=1}^{\infty} \frac{\left|f_{a}^{\prime}\left(x_{a, j}\right)-f_{b}^{\prime}\left(y_{b, j}\right)\right|}{\left|f_{b}^{\prime}\left(y_{b, j}\right)\right|}<\infty
$$

which suffices to get bounded distortion as announced.

For future reference let us state what we have just proved.

Proposition 4.3. Let $\omega \subset \mathcal{P}_{n}$, satisfying $(B A)_{j}$ and $(E G)_{j}$ for $j=1, \ldots, n$. Let $\Delta \leq$ $|m| \leq[\alpha n]$ and write $p=p(m, \omega)$. Then, there is a constant $C_{1}=C_{1}(\alpha)$ such that for all $a \in \omega$ we have:

(a) $\frac{1}{C_{1}} \leq \frac{\left|\left(f_{a, \theta}^{j}\right)^{\prime}\left(f_{a, \theta}(x)\right)\right|}{\left|\left(f_{a, \theta}^{j}\right)^{\prime}\left(f_{a, \theta}(a)\right)\right|} \leq C_{1} \quad$ for $j=1, \ldots, p$ and every $x \in U_{m}^{+}(\omega)$;
(b) $p<50|m| ;$
(c) $\left|\left(f_{a, \theta}^{p+1}\right)^{\prime}(x)\right| \geq e^{(1-50 \beta)|m|}$ for every $x \in I_{m}^{+}(\omega)$.

Remark 4.4. Note that items (b) and (c) above imply that if $\Delta$ is sufficiently large so that $|m| \geq \Delta$ implies $10 \beta|m|>1$ then

$$
\left|\left(f_{a, \theta}^{p+1}\right)^{\prime}(x)\right| \geq e^{(1-60 \beta) p+1} \quad \text { for all } x \in I_{m}^{+}(\omega) .
$$

Another piece of useful information about the dynamics of parameter intervals is a comparison between the sizes of its iterates in different moments. This is the content of the next lemma.

LEMmA 4.5. Let $\omega \in \mathcal{P}_{n}$ and $i<j \leq n$ where $\left|\xi_{n}(\omega)\right|<1$. Then, for some $t \in \xi_{i}(\omega)$, we have

$$
\frac{1}{Q^{2}}\left|\left(f_{t}^{j-i}\right)^{\prime}(t)\right| \leq \frac{\left|\xi_{j}(\omega)\right|}{\left|\xi_{i}(\omega)\right|} \leq Q^{2}\left|\left(f_{t}^{j-i}\right)^{\prime}(t)\right|
$$

Proof. We choose arbitrarily $a, b \in \omega$ and write $a_{j}=f_{a}^{j}(a)$ and $b_{j}=f_{b}^{j}(b)$. Since by induction hypothesis we have expansion up to $n$ and $\xi_{n}(\omega)$ does not cover $\mathbb{S}^{1}$ then $\xi_{i}: \omega \rightarrow \xi_{i}(\omega)$ and $\xi_{j}: \omega \rightarrow \xi_{j}(\omega)$ are diffeomorphisms. Thus,

$$
\left|a_{j}-b_{j}\right|=\left|\xi_{j} \circ \xi_{i}^{-1}\left(a_{i}\right)-\xi_{j} \circ \xi_{i}^{-1}\left(b_{i}\right)\right| .
$$

By the mean value theorem, there exists $t$ between $\xi(a)$ and $\xi(b)$ such that

$$
\left|a_{j}-b_{j}\right|=\left|\left(\xi_{j} \circ \xi_{i}^{-1}\right)^{\prime}(t)\right|\left|a_{i}-b_{i}\right|=\frac{\left|\xi_{j}^{\prime}\left(\xi_{i}^{-1}(t)\right)\right|}{\left|\xi_{i}^{\prime}(t)\right|}\left|a_{i}-b_{i}\right| .
$$

Since $a, b \in \omega$ are taken arbitrarily, the claim follows from Lemma 2.1. 


\section{Parameter exclusions}

In order to estimate the measure of the subset of excluded parameters we have to deal with the size of the iterates of the intervals we are following as well as the frequency for which these returns are well sized.

This is accomplished by considering the components of intervals $\omega$ that are mapped to extremes of the critical region. More precisely, consider $\omega \in \mathcal{P}_{n-1}$ and its associated partition $\left\{I_{m, k}\right\}_{m, k}$. Suppose that $n$ is a return time for $\omega$. If $\omega^{\prime} \subset \omega$ has a host interval satisfying $m=\Delta-1$ then we call $n$ an escape time for all $a \in \omega^{\prime}$ and the interval $\omega^{\prime}$ itself will be referred to as an escape component.

The next lemma states that an escape component will return large. This implies that new escape components will be created with high probability.

Lemma 5.1. Let $\omega \in \mathcal{P}_{n}$ be an escape component and $\mu$ its next return time. Then, for some $\rho>0$,

$$
\left|\xi_{\mu}(\omega)\right| \geq e^{-\rho \Delta}
$$

Proof. We can suppose $\left|\xi_{n}(\omega)\right|<1$ since otherwise the claim is obvious.

Recall that $\Delta+1=-\log \delta$ and note that $\tilde{\delta}=\omega_{+}+\delta$ or $\tilde{\delta}=\omega_{-}-\delta$ belongs to $\xi_{n}(\omega)$. In order to simplify our proof let us consider the first case since the other one is similar.

Let $a \in \mathcal{P}_{n}$ be a parameter for which $\xi_{n}(a)=\tilde{\delta}$. Then, using the local form (1), we have

$$
\left|f_{a}(\tilde{\delta})-f_{a}(a)\right| \leq-A_{2} \theta(\delta+|\omega|)+A_{4}(\delta+|\omega|)^{3} .
$$

Let $p_{a}$ be the fixed point of $f_{a}$ which is the analytical continuation of $p$. From (10) we have

$$
\begin{aligned}
\left|f_{a}(\tilde{\delta})-p_{a}\right| & \leq\left|f_{a}(\tilde{\delta})-f_{a}(a)\right|+\left|f_{a}(a)-p_{a}\right| \\
& \leq-2 A_{2} \theta \delta+2 A_{4} \delta^{3}+\delta^{3} .
\end{aligned}
$$

Since $-\theta \leq \delta^{2}$, for some constant $C_{0}>0$, we have

$$
\left|f_{a}(\tilde{\delta})-p_{a}\right| \leq C_{0} \delta^{3} .
$$

Hence, if $M=\sup \left\{f_{a}^{\prime}(x)\right\}$, then

$$
\left|f_{a}^{k}(\tilde{\delta})-f_{a}^{k}\left(p_{a}\right)\right| \leq M^{k-1}\left|f_{a}(\tilde{\delta})-p_{a}\right| \leq C_{0} M^{k-1} \delta^{3} .
$$

Therefore, taking $C=C_{0} M^{-1}$,

$$
\left|f_{a}^{\mu-n}(\tilde{\delta})-p_{a}\right| \leq C M^{\mu-n} \delta^{3} .
$$

If we suppose that the next return $\mu$ occurs while

$$
C M^{\mu-n} \delta^{3}<\frac{1}{4}
$$

then $\xi_{\mu}(\omega)$ has an extremal point, $f_{a}^{\mu-n}(\tilde{\delta})$, far from the return region, and contains some point inside the return region. Otherwise,

$$
C M^{\mu-n} \delta^{3} \geq \frac{1}{4}
$$


implies that

$$
\mu-n \geq \frac{-\log \left(4 C \delta^{3}\right)}{\log M} .
$$

Moreover, by Proposition 4.3 and Lemma 4.5, we have

$$
\frac{\left|\xi_{\mu}(\omega)\right|}{\left|\xi_{n}(\omega)\right|} \geq Q^{-2}\left|\left(f_{a}^{\mu-n}\right)^{\prime}\left(\xi_{n}(a)\right)\right| \geq Q^{-2} C_{1}\left|\left(f_{a}^{\mu-n}\right)^{\prime}\left(\xi_{n}(\tilde{\delta})\right)\right| .
$$

Since $n$ is an escape time for $\omega$, we have $\xi_{n}(\omega)$ outside $(a-\delta, a+\delta)$ and so, by Proposition 2.3, all orbits starting at $\xi_{n}(\omega)$ expand uniformly from a return situation to the next one. Using Lemma 2.1 the same holds on parameter space. Thus,

$$
\frac{\left|\xi_{\mu}(\omega)\right|}{\left|\xi_{n}(\omega)\right|} \geq C_{1} Q^{-2} e^{c(\mu-n)} .
$$

By hypothesis, $n$ is an escape time for $\omega$. Then

$$
\left|\xi_{n}(\omega)\right| \geq \frac{e^{-\Delta}}{\Delta^{2}}
$$

Therefore from (23) and (24), there exists a constant $\rho>0$ such that (recall $e^{-(\Delta+1)}=\delta$ )

$$
\begin{aligned}
\left|\xi_{\mu}(\omega)\right| & \geq C_{1} Q^{-2} e^{-c\left(\log \left(4 C \delta^{3}\right) / \log M\right)}\left|\xi_{n}(\omega)\right| \\
& \geq C_{1} Q^{-2} e^{-c\left(\log \left(4 C \delta^{3}\right) / \log M\right)} \frac{e^{-\Delta}}{\Delta^{2}} \\
& \geq \tilde{C} e^{3 c(\Delta+1) / \log M} \frac{e^{-\Delta}}{\Delta^{2}} \\
& \geq \tilde{C} \frac{e^{((3 c-1) / \log M) \Delta}}{\Delta^{2}} \geq e^{-\rho \Delta},
\end{aligned}
$$

where $\tilde{C}$ is a positive constant.

The next lemma points toward an exponentially fast growth of the intervals of the partition $\mathcal{P}_{n}$. In fact, it provides the essential estimates that enable us to reach that conclusion.

LEMMA 5.2. Let $\mu$ be a return for $\omega \in \mathcal{P}_{n}$ with host interval $I_{m, k}$ and $p$ its binding period. Then, if $\mu^{\prime} \leq n$ is the next return after $\mu$ and $q=\mu^{\prime}-\mu-p-1$, we have:

(a) $\left|\xi_{\mu^{\prime}}(\omega)\right| \geq e^{c q} e^{(1-60 \beta)|m|}\left|\xi_{\mu}(\omega)\right| \geq 2\left|\xi_{\mu}(\omega)\right|$;

(b) $\left|\xi_{\mu^{\prime}}(\omega)\right| \geq e^{c q} e^{-60 \beta|m|}$, if $\mu$ is an essential return.

Proof. Write

$$
\frac{\left|\xi_{\mu^{\prime}}(\omega)\right|}{\left|\xi_{\mu}(\omega)\right|}=\frac{\left|\xi_{\mu^{\prime}}(\omega)\right|}{\left|\xi_{\mu+p+1}(\omega)\right|} \frac{\left|\xi_{\mu+p+1}(\omega)\right|}{\left|\xi_{\mu}(\omega)\right|}
$$

Then, from Lemma 4.5 and Proposition 4.3, we get

$$
\begin{aligned}
\frac{\left|\xi_{\mu^{\prime}}(\omega)\right|}{\left|\xi_{\mu}(\omega)\right|} & \geq Q^{-4}\left|\left(f_{t}^{q}\right)^{\prime}\left(\xi_{\mu+p+1}(t)\right)\right|\left|\left(f_{t^{\prime}}^{p+1}\right)^{\prime}\left(\xi_{\mu}\left(t^{\prime}\right)\right)\right| \\
& \geq Q^{-4}\left|\left(f_{t}^{q}\right)^{\prime}\left(\xi_{\mu+p+1}(t)\right)\right| e^{(1-50 \beta)|m|} .
\end{aligned}
$$


Part (a) follows from

$$
\left|\left(f_{t}^{q}\right)^{\prime}\left(\xi_{\mu+p+1}(t)\right)\right| \geq e^{c q} .
$$

Furthermore, if $\mu$ is an essential return then part (b) is a consequence of

$$
\left|\xi_{\mu}(\omega)\right| \geq \frac{e^{-|m|}}{m^{2}} .
$$

Remark 5.3. It follows from the preceding lemma and the induction hypothesis (FA) ${ }_{n}$ that if $\mu$ is a return time for $\omega \in \mathcal{P}_{n}$ then

$$
\left|\xi_{\mu}(\omega)\right| \geq e^{c(1-\alpha) \mu}|\omega| .
$$

On returns we prove that $\left|\xi_{\mu}(\omega)\right|$ is very large when compared with $\left|U_{[\alpha n]}\right|$ which is an important ingredient to estimate exclusions due to the $(\mathrm{BA})_{n}$ condition.

Lemma 5.4. Suppose $n$ is a return time for $\omega \in \mathcal{P}_{n-1}$. Then

$$
\left|\xi_{n}(\omega)\right| \geq e^{-\alpha n / 2} .
$$

Proof. Take the greatest $\mu \leq n-1$ satisfying $\omega_{\mu}=\omega$. Then $\xi_{\mu}(\omega)$ contains some host interval $I_{m, k}$ by construction. If we suppose that $\mu$ is an escape time, that is, $|m|=\Delta-1$, then, from Lemma 5.1, $\left|\xi_{n}(\omega)\right| \geq e^{-\rho \Delta} \geq e^{-\alpha n / 2}$ if $n$ is large enough.

Otherwise, due to the (BA) $)_{n}$ condition, $|\alpha n-1| \geq|m| \geq \Delta$. Let $\mu_{0}=\mu, \mu_{s+1}=n$ and $\left(\mu_{i}\right)_{i=1, \ldots, s}$ the returns of $\omega$ after $\mu$. Then, from Lemma 5.2, we have

$$
\left|\xi_{n}(\omega)\right| \geq\left|\xi_{\mu_{1}}(\omega)\right| \prod_{i=1}^{s-1} \frac{\left|\xi_{\mu_{i+1}}(\omega)\right|}{\left|\xi_{\mu_{i}}(\omega)\right|} \frac{\left|\xi_{\mu_{s+1}}(\omega)\right|}{\left|\xi_{\mu_{s}}(\omega)\right|} \geq e^{-60 \beta|m|} 2^{s-1}
$$

Therefore,

$$
\left|\xi_{n}(\omega)\right| \geq e^{-60 \beta(\alpha n)} \geq e^{-\alpha n / 2} .
$$

PROPOSITION 5.5. There exists a constant $C$ depending only on $\alpha$ such that if $\omega \in \mathcal{P}_{n-1}$ and $n$ is a return time for $\omega$ then for all $a, b \in \omega$ we have

$$
\frac{\left|\left(f_{a}^{n}\right)^{\prime}(a)\right|}{\left|\left(f_{b}^{n}\right)^{\prime}(b)\right|} \leq C \quad \text { and } \quad \frac{\left|\xi_{n}^{\prime}(a)\right|}{\left|\xi_{n}^{\prime}(b)\right|} \leq C .
$$

Proof. By Lemma 2.1 it is suffices to prove the left inequality.

Denote by $\mu_{i}, i=1, \ldots, s$, the returns of $\omega$ up to time $n$ with corresponding bound periods of length $p_{i}$, host intervals $I_{m_{1}, k_{1}}, \ldots, I_{m_{s}, k_{s}}$ and $\sigma_{i}=\xi_{\mu_{i}}(\omega)$. Let $\mu_{0}=1$, $p_{0}=-1$.

We have

$$
\frac{\left(f_{a}^{n}\right)^{\prime}(a)}{\left(f_{b}^{n}\right)^{\prime}(b)}=\prod_{j=0}^{n-1} \frac{f_{a}^{\prime}\left(f_{a}^{j}(a)\right)}{f_{b}^{\prime}\left(f_{b}^{j}(b)\right)}=\prod_{j=0}^{n-1}\left(1+\frac{f_{a}^{\prime}\left(f_{a}^{j}(a)\right)-f_{b}^{\prime}\left(f_{b}^{j}(b)\right)}{f_{b}^{\prime}\left(f_{b}^{j}(b)\right)}\right) .
$$

Hence, it is sufficient to show that

$$
\sum_{j=0}^{\infty} \frac{f_{a}^{\prime}\left(f_{a}^{j}(a)\right)-f_{b}^{\prime}\left(f_{b}^{j}(b)\right)}{f_{b}^{\prime}\left(f_{b}^{j}(b)\right)}<\infty .
$$


Let us write $a_{j}=f_{a}^{j}(a)$ and $b_{j}=f_{b}^{j}(b)$. The fact that $f_{a}(x)$ is a $C^{2}$-map implies that there exist $t \in[a, b] \subset \omega$ and $z_{j}$ between $a_{j}$ and $b_{j}$ such that

$$
\begin{aligned}
S_{j}=\frac{\left|f_{a}^{\prime}\left(a_{j}\right)-f_{b}^{\prime}\left(b_{j}\right)\right|}{\left|f_{b}^{\prime}\left(b_{j}\right)\right|} & =\frac{\left|f_{a}^{\prime}\left(a_{j}\right)-f_{a}^{\prime}\left(b_{j}\right)+f_{a}^{\prime}\left(b_{j}\right)-f_{b}^{\prime}\left(b_{j}\right)\right|}{\left|f_{b}^{\prime}\left(b_{j}\right)\right|} \\
& \leq \frac{\left|f_{a}^{\prime \prime}\left(z_{j}\right)\right|\left|a_{j}-b_{j}\right|+\left|\partial_{a} f_{t}^{\prime}\left(b_{j}\right)\right||a-b|}{\left|f_{b}^{\prime}\left(b_{j}\right)\right|} .
\end{aligned}
$$

For estimating $\sum S_{j}$ on return times, we assume that $j=\mu_{i}$ for some $i=1, \ldots, s$. Then $b_{j} \in I_{m_{i}, k_{i}}$ and so the derivative $\left|f_{b}^{\prime}\left(b_{j}\right)\right| \geq\left(e^{-\left|m_{i}\right|}\right)^{2} \geq\left|I_{m_{i}}\right|^{2}$. On the other hand, the factors $\left|f_{a}^{\prime \prime}\left(z_{j}\right)\right|$ and $\left|\partial_{a} f_{t}^{\prime}\left(b_{j}\right)\right|$ are bounded simultaneously by $C\left|I_{m_{i}}\right|$ for some constant $C$. Furthermore by Lemma 5.2 the size of $\xi_{j}(\omega)$ gets expanded between returns and so $|a-b|<\left|a_{j}-b_{j}\right|$ for all $j$. Hence we can write

$$
S_{\mu_{i}} \leq \frac{C\left|I_{m_{i}}\right|\left|a_{j}-b_{j}\right|}{\left|I_{m_{i}}\right|^{2}} \leq \frac{C \sigma_{i}}{\left|I_{m_{i}}\right|}
$$

We group the remaining terms of $\sum S_{j}$ taking into account the bound periods and free times. One considers the partial sums for $i=0, \ldots, s-1$,

$$
S_{i}^{b}=\sum_{j=\mu_{i}+1}^{\mu_{i}+p_{i}} S_{j} \quad \text { and } \quad S_{i}^{f}=\sum_{j=\mu_{i}+p_{i}+1}^{\mu_{i+1}-1} S_{j} .
$$

Let us estimate $S_{i}^{f}$ for $i=0, \ldots, s-1$ and $\mu_{i}+p_{i}+1 \leq j \leq \mu_{i+1}$. Again by Lemma 5.2 we have $|a-b|<\left|a_{j}-b_{j}\right|$ for all such index $j$. Additionally we have a uniform bound $K$ for $\left|f_{a}^{\prime \prime}\left(z_{j}\right)\right|+\left|\partial_{a} f_{t}^{\prime}\left(b_{j}\right)\right|$ and so

$$
S_{j} \leq K \frac{\left|a_{j}-b_{j}\right|}{\left|f_{b}^{\prime}\left(b_{j}\right)\right|} .
$$

From Lemma 4.5 there exists $t_{j} \in \omega$ such that

$$
\frac{\left|a_{\mu_{i}+1}-b_{\mu_{i}+1}\right|}{\left|a_{j}-b_{j}\right|} \geq \frac{1}{Q^{2}}\left|\left(f_{t_{j}}^{\mu_{i+1}-j}\right)^{\prime}\left(\xi_{j}\left(t_{j}\right)\right)\right| \geq Q^{-2} e^{c\left(\mu_{i+1}-j\right)},
$$

where the last inequality is due to the fact that $j$ is a free time. Therefore, since $j$ is a free time (and then $\left|f_{b}^{\prime}\left(b_{j}\right)\right| \geq M \delta^{2}$ ), we have

$$
\frac{\left|a_{j}-b_{j}\right|}{\left|f_{b}^{\prime}\left(b_{j}\right)\right|} \leq \frac{Q^{2}}{M \delta^{2}} e^{-c\left(\mu_{i+1}-j\right)}\left|\sigma_{i+1}\right|
$$

Hence, there exists a positive constant $\tilde{C}_{0}$ such that

$$
S_{i}^{f} \leq K \frac{Q^{2}}{\delta^{2}}\left|\sigma_{i+1}\right| \sum_{j=\mu_{i}+p_{i}+1}^{\mu_{i+1}-1} e^{c\left(j-\mu_{i+1}\right)} \leq \tilde{C}_{0}\left|I_{m_{i+1}}\right| \frac{\left|\sigma_{i+1}\right|}{\left|I_{m_{i+1}}\right|} \leq c_{f} \frac{\left|\sigma_{i+1}\right|}{\left|I_{m_{i+1}}\right|},
$$

for some positive constant $c_{f}$.

In order to estimate $S_{i}^{b}$ we first observe that $\mu_{i}+1 \leq j \leq \mu_{i}+p_{i}$ and use Remarks 2.4 and 5.3 to get

$$
\left|a_{j}-b_{j}\right| \geq \delta^{2} e^{-2\left(\mu_{i}+1\right)}\left|a_{\mu_{i}}-b_{\mu_{i}}\right| \geq \delta^{2} e^{-2\left(\mu_{i}+1\right)} e^{c(1-\alpha) \mu_{i}}|a-b| .
$$


Hence $\left|a_{j}-b_{j}\right| \geq|a-b|$ and we have the same bound for $S_{j}$ as given in (26).

We write

$$
\frac{\left|a_{j}-b_{j}\right|}{\left|f_{b}^{\prime}\left(b_{j}\right)\right|}=\frac{\left|a_{j}-b_{j}\right|}{\left|b_{j}-b_{j-\mu_{i}}\right|} \frac{\left|b_{j}-b_{j-\mu_{i}}\right|}{\left|f_{b}^{\prime}\left(b_{j}\right)\right|} .
$$

From the definition of bound period we have

$$
\left|b_{j}-b_{j-\mu_{i}}\right| \leq e^{-\beta\left(j-\mu_{i}\right)} .
$$

Moreover, as $\left|f_{b}^{\prime}\left(b_{j}\right)\right| \geq M\left|b_{j}-b\right|^{2}$ and

$$
\begin{aligned}
\left|b_{j}-b\right| & \geq\left|b_{j-\mu_{i}}-b+b_{j}-b_{j-\mu_{i}}\right| \geq\left|b_{j-\mu_{i}}-b\right|-e^{-\beta\left(j-\mu_{i}\right)} \\
& \geq e^{-\alpha\left(j-\mu_{i}\right)}-e^{-\beta\left(j-\mu_{i}\right)} \geq\left(1-e^{-(\beta-\alpha)}\right) e^{-\alpha\left(j-\mu_{i}\right)} .
\end{aligned}
$$

Hence, if $M_{1}=\left(M\left(1-e^{-(\beta-\alpha)}\right)^{2}\right)^{-1}$, then

$$
\frac{\left|b_{j}-b_{j-\mu_{i}}\right|}{\left|f_{b}^{\prime}\left(b_{j}\right)\right|} \leq M_{1} e^{-(\beta-2 \alpha)\left(j-\mu_{i}\right)} .
$$

In order to bound the other factor in (27), we use Lemma 4.5 to get, for some $t \in \omega$,

$$
\frac{\left|a_{j}-b_{j}\right|}{\left|a_{\mu_{i}}-b_{\mu_{i}}\right|} \leq Q^{2}\left|\left(f_{t}^{j-\mu_{i}-1}\right)^{\prime}\left(f_{t}\left(t_{\mu_{i}}\right)\right)\right|\left|f_{t}^{\prime}\left(t_{\mu_{i}}\right)\right| .
$$

There exists $x \in U_{m}^{+}$such that

$$
\left|b_{j}-b_{j-\mu_{i}}\right|=\left|\left(f_{b}^{j-\mu_{i}-1}\right)^{\prime}\left(f_{b}(x)\right)\right|\left|f_{b}\left(b_{\mu_{i}}\right)-f_{b}(b)\right| \text {. }
$$

From local forms (1) and (2), we have

$$
\left|f_{t}^{\prime}\left(t_{\mu_{i}}\right)\right| \leq-\theta A_{2}+3 A_{4}\left(t_{\mu_{i}}-t\right)^{2}
$$

and

$$
\begin{aligned}
\left|f_{b}\left(b_{\mu_{i}}\right)-f_{b}(b)\right| & \geq-\theta A_{1}\left|b_{\mu_{i}}-b\right|+A_{3}\left|b_{\mu_{i}}-b\right|^{3} \\
& =\left|b_{\mu_{i}}-b\right|\left(-\theta A_{1}+A_{3}\left(b_{\mu_{i}}-b\right)^{2}\right) .
\end{aligned}
$$

Thus, for $\theta \leq 0$, we have

$$
\begin{aligned}
\frac{\left|f_{t}^{\prime}\left(t_{\mu_{i}}\right)\right|}{\left|f_{b}\left(b_{\mu_{i}}\right)-f_{b}(b)\right|} & \leq \frac{1}{\left|b_{\mu_{i}}-b\right|} \frac{-\theta A_{2}+3 A_{4}\left(t_{\mu_{i}}-t\right)^{2}}{-\theta A_{1}+A_{3}\left(b_{\mu_{i}}-b\right)^{2}} \\
& =\frac{1}{\left|b_{\mu_{i}}-b\right|}\left(\frac{-\theta A_{2}}{-\theta A_{1}+A_{3}\left(b_{\mu_{i}}-b\right)^{2}}+\frac{3 A_{2}\left(t_{\mu_{i}}-t\right)^{2}}{-\theta A_{1}+A_{3}\left(b_{\mu_{i}}-b\right)^{2}}\right) \\
& \leq \frac{1}{\left|b_{\mu_{i}}-b\right|}\left(\frac{A_{2}}{A_{1}}+\frac{4 A_{4}\left|t_{\mu_{i}}-t\right|^{2}}{A_{3}\left|b_{\mu_{i}}-b\right|^{2}}\right),
\end{aligned}
$$

whenever $\theta$ is sufficiently small. Since $\mu_{i}$ is a return time for $\omega, b_{\mu_{i}}$ and $t_{\mu_{i}}$ belong to the same host interval $I_{m_{i}, k}$. The distance from $I_{m_{i}, k}$ to $\omega$ is greater than $e^{-m+1}$ and less than $e^{-m}+|\omega|$. Hence, there is a positive constant $A$ such that

$$
\frac{\left|f_{t}^{\prime}\left(t_{\mu_{i}}\right)\right|}{\left|f_{b}\left(b_{\mu_{i}}\right)-f_{b}(b)\right|} \leq \frac{A}{\left|b_{\mu_{i}}-b\right|} \text {. }
$$


Thus, from (28) and (30) we have

$$
\frac{\left|a_{j}-b_{j}\right|}{\left|f_{b}\left(b_{\mu_{i}}\right)-f_{b}(b)\right|} \leq A Q^{2}\left|\left(f_{t}^{j-\mu_{i}-1}\right)^{\prime}\left(f_{t}\left(t_{\mu_{i}}\right)\right)\right| \frac{\left|a_{\mu_{i}}-b_{\mu_{i}}\right|}{\left|b_{\mu_{i}}-b\right|} .
$$

Therefore, from using (29) and (31) we obtain

$$
\frac{\left|a_{j}-b_{j}\right|}{\left|b_{j}-b_{j-\mu_{i}}\right|} \leq A Q^{2} \frac{\left|\left(f_{t}^{j-\mu_{i}-1}\right)^{\prime}\left(f_{t}\left(t_{\mu_{i}}\right)\right)\right|}{\left|\left(f^{j-\mu_{i}-1}\right)^{\prime}\left(f_{b}(x)\right)\right|} \frac{\left|a_{\mu_{i}}-b_{\mu_{i}}\right|}{\left|b_{\mu_{i}}-b\right|} .
$$

Since $f_{t}\left(t_{\mu_{i}}\right)$ and $f_{b}(x)$ are far from the perturbation region, we conclude that there is a positive constant $A_{0}$ such that

$$
\frac{\left|a_{j}-b_{j}\right|}{\left|b_{j}-b_{j-\mu_{i}}\right|} \leq A_{0} \frac{\left|a_{\mu_{i}}-b_{\mu_{i}}\right|}{\left|b_{\mu_{i}}-b\right|} .
$$

Hence,

$$
S_{i}^{b} \leq c_{b} \frac{\left|\sigma_{i}\right|}{\left|I_{m_{i}}\right|}
$$

for some positive constant $c_{b}$.

Thus, there exists a positive constant $C$, such that

$$
S_{i-1}^{f}+S_{\mu_{i}}+S_{i}^{b} \leq C \frac{\left|\sigma_{i}\right|}{\left|I_{m_{i}}\right|} .
$$

The fact that $\left|\sigma_{j+1}\right| \geq 2\left|\sigma_{j}\right|$ implies that, for each fixed $m$, the sequence of all $\left|\sigma_{m_{i}}\right|, \sigma_{m_{i}} \subset I_{m}$, is geometrically increasing. Hence, the sum of $\left|\sigma_{m_{i}}\right| /\left|I_{m}\right|$ over the corresponding values of $i$ is bounded by a multiple of the last term:

$$
\sum_{i=1}^{s} \frac{\left|\sigma_{i}\right|}{\left|I_{m_{i}}\right|}=\sum_{m>0} \sum_{i: m_{i}=m} \frac{\left|\sigma_{i}\right|}{\left|I_{m}\right|} \leq \sum_{m>0} \frac{\left|\sigma_{i(m)}\right|}{\left|I_{m}\right|},
$$

where, by definition, $i(m)$ is the largest value of $i$ for which $m_{i}=m$. By construction, $\sigma_{i}$ belongs to some $I_{m, k}^{+}$. Then, there is a positive constant $C_{0}$ such that

$$
\frac{\left|\sigma_{i(m)}\right|}{\left|I_{m}\right|} \leq \frac{\left|I_{m, k}^{+}\right|}{\left|I_{m}\right|} \leq \frac{C_{0}}{m^{2}}
$$

Therefore,

$$
S=\sum_{j=1}^{s} S_{i-1}^{f}+S_{\mu_{i}}+S_{i}^{b} \leq C \sum_{i=1}^{s} \frac{\left|\sigma_{i}\right|}{\left|I_{m_{i}}\right|} \leq C_{0} \sum_{m>0} \frac{1}{m^{2}}<\infty .
$$

and the proof of Proposition 5.5 is complete.

\section{Estimating parameter exclusions}

Recall that $\Omega_{n}$ is the set of the remaining parameters at stage $n$. Let us write $\Omega_{n+1}^{\prime}$ for the set of parameters just obtained by imposing the (BA) $)_{n}$ condition to $\Omega_{n}$ as described before.

LEMMA 6.1. For $n$ sufficiently large it holds that

$$
\operatorname{Leb}\left(\Omega_{n} \backslash \Omega_{n+1}^{\prime}\right) \leq e^{-\varepsilon n} \operatorname{Leb}\left(\Omega_{n}\right),
$$

for some fixed $\varepsilon>0$. 
Proof. Suppose $n$ is a return time for $\omega$ when we exclude an interval $\bar{\omega}$ imposing the (BA) $n$ restriction. Then $\left|\xi_{n}(\bar{\omega})\right| \leq 2 e^{-\alpha n+1}$.

Therefore

$$
\frac{|\bar{\omega}|}{|\omega|} \leq \frac{\left|\xi_{n}^{\prime}(t)\right|\left|\xi_{n}(\bar{\omega})\right|}{\left|\xi_{n}^{\prime}(\bar{t})\right|\left|\xi_{n}(\omega)\right|}
$$

for some $t \in \omega$ and $\bar{t} \in \bar{\omega}$.

Using the bounded distortion given by Proposition 5.5 and Lemma 5.4, we have

$$
\frac{|\bar{\omega}|}{|\omega|} \leq \frac{2 C e^{1-\alpha n}}{e^{-\alpha n / 2}}=2 C e^{1-\alpha n / 2} \leq e^{-\alpha n / 3},
$$

whenever $n$ is sufficiently large. This implies the statement.

Since we construct $\Omega_{n+1}$ from $\Omega_{n+1}^{\prime}$ by imposing the (FA) $n$ condition, all that remains to be proved is that $\operatorname{Leb}\left(\Omega_{n+1}^{\prime} \backslash \Omega_{n+1}\right)$ decreases exponentially fast with $n$. We use a large deviation argument that can be carried out exactly in the same way as in $[\mathbf{2}, \mathbf{3}, \mathbf{1 3}]$ as soon as we have some basic results. Among these facts, we have already established estimates for the growth of $\left|\xi_{n}(\omega)\right|$ between returns (see Lemma 5.2). Now we turn our attention to the frequency of returns. This is motivated by the following considerations. The assumption $(\mathrm{FA})_{n}$ is related to the amount of time the orbits remain inside free periods. Good trajectories are those that spend a small proportion of time recovering losses of derivative. Among the iterates which contribute with real expansion, we have those pieces of orbits which are escape periods, that is to say, those iterates between successive escape times. In order to estimate the total amount of time spent in escape periods, we need to estimate the expected time between escape situations. This can be carried out if we can relate the length of orbit between successive returns to the distance of a return to the critical point. This is the content of the next lemma.

LemMA 6.2. Suppose $n$ is an essential return for the interval $\omega \in \mathcal{P}_{n}$ with $I_{m, k} \subset \xi_{n}(\omega)$. Then the next return situation $v$ satisfies

$$
v<n+80|m| \text {. }
$$

Proof. In order to simplify notations we suppose $m>0$. First of all, let $n=\mu_{0}<$ $\mu_{1}<\cdots<\mu_{s}<\mu_{s+1}=v$ be the intermediate inessential returns with host intervals $I_{m_{i}, k_{i}}$. Moreover, for all $i=0, \ldots, s$, we write $p_{i}$ for the bound period and $q_{i}=\mu_{i+1}-\left(\mu_{i}+p_{i}+1\right)$ for the free period. Again, we write simply $m_{i}$ instead of $\left|m_{i}\right|$.

By Lemma 5.2, we have

$$
\left|\xi_{\mu_{1}}(\omega)\right| \geq e^{-60 \beta m} e^{c q_{0}}
$$

and

$$
\frac{\left|\xi_{i+1}(\omega)\right|}{\left|\xi_{i}(\omega)\right|} \geq e^{c q_{i}} e^{(1-60 \beta) m_{i}}
$$

Therefore,

$$
\left|\xi_{\mu_{s+1}}(\omega)\right|=\left|\xi_{\mu_{1}}(\omega)\right| \prod_{i=1}^{s} \frac{\left|\xi_{\mu_{i+1}}(\omega)\right|}{\left|\xi_{\mu_{i}}(\omega)\right|} \geq e^{-60 \beta m} e^{c q_{0}} \prod_{i=1}^{s} e^{c q_{i}} e^{(1-60 \beta) m_{i}}
$$


Now, observe that we have a natural bound

$$
\left|\xi_{\mu_{s+1}}(\omega)\right| \leq \operatorname{deg}(f) .
$$

By taking $\Delta$ sufficiently large we can suppose $m \gg \operatorname{deg}(f)(m \geq \Delta)$. Taking logarithms above, we get

$$
\sum_{i=0}^{s} c q_{i}+\sum_{i=1}^{s}(1-60 \beta) m_{i} \leq \log (\operatorname{deg}(f))+60 \beta m .
$$

Furthermore, since we have $c>1 / 30$, then,

$$
v-n=\sum_{i=0}^{s}\left(p_{i}+q_{i}+1\right) \leq \sum_{i=0}^{s}\left(p_{i}+60 c q_{i}\right) .
$$

But, from Proposition 4.3, we have

$$
p_{i} \leq 50 m_{i} \leq 60(1-60 \beta) m_{i} .
$$

Therefore, from (32),

$$
\begin{aligned}
v-n & \leq 60 m+60\left(\sum_{i=0}^{s} c q_{i}+\sum_{i=1}^{s}(1-60 \beta) m_{i}\right) \\
& \leq 60 m+60(\log (\operatorname{deg}(f))+60 \beta m) \leq 80 m .
\end{aligned}
$$

After this result we can follow the same standard large deviation arguments presented in [3] and finally get

$$
\operatorname{Leb}\left(\left\{a \in \Omega_{n+1}^{\prime}: F_{n}(a) \leq(1-\alpha) n\right\}\right) \leq e^{-\epsilon n} \operatorname{Leb}\left(\Omega_{0}\right)
$$

for some fixed $\epsilon>0$.

Finally we get the following result.

PROPOSITION 6.3. The set $\Omega_{\theta}$ has positive Lebesgue measure.

Proof. The proof is a direct consequence of (33) and Lemma 6.1, taking into account that exclusions on $\Omega_{0}$ may occur only after $N$ iterates (recall that $N \gg 1$ ). So we can assume that $\Omega_{0}=\cdots=\Omega_{N}$ and $n \geq N$ in the two preceding lemmas.

\section{Growth of derivatives}

Proposition 7.1. For each fixed negative $\theta$ close to zero there exist $\hat{c}=\hat{c}(\theta)>0$ and $\sigma_{1}>1$ such that, for all $a \in \Omega_{\theta}$ and $k \in \mathbb{N}$, we have, for every $x \in \mathbb{S}^{1}$,

$$
\left|\left(f_{a}^{k}\right)^{\prime}(x)\right| \geq \hat{c} \sigma_{1}^{k} .
$$

Proof. Let $\theta<0$ and $a \in \Omega_{\theta}$ be fixed and write $f=f_{a, \theta}$. Given $x \in \mathbb{S}^{1}$ we put $x_{0}=x$ and $x_{j}=f\left(x_{j-1}\right)$, for all $j \geq 1$. Let us introduce the following notation:

$$
D_{k, n}(x)=\prod_{j=k}^{n}\left|f^{\prime}\left(x_{j}\right)\right| \text { and } \quad D_{n}(x)=\prod_{j=0}^{n}\left|f^{\prime}\left(x_{j}\right)\right| .
$$


We can extend, in a natural way, to the orbit of $x$ the notions of return time and bound period of the critical orbit.

For every $x \in \mathbb{S}^{1}$ such that $f^{\prime}\left(x_{n}\right) \neq 0$ for all $n \geq 0$, let $\left(n_{k}\right)_{k}$ be the sequence of return times of $x$ by the map $f$ with associated bound periods $\left(p_{k}\right)_{k}$. Since during the bound period the orbit of $x$ inherits the same expanding behavior of the critical orbit (Proposition 4.3, part (a)), we have

$$
D_{n_{k}, n_{k}+j}(x) \geq\left|f^{\prime}\left(x_{n_{k}}\right)\right| C_{1}^{-1} e^{c j}, \quad 0 \leq j<p_{k} .
$$

At the end of the bound period, Remark 4.4 gives

$$
D_{n_{k}, n_{k}+p_{k}}(x) \geq e^{(1-60 \beta) p_{k}+1} .
$$

Using Proposition 2.3 and Remark 2.4, we get

$$
D_{n_{k}, n_{k}+p_{k}+j}(x) \geq \sigma_{1}^{p_{k}+1} \delta^{2} \sigma_{1}^{j} \quad \text { for all } 1 \leq j<n_{k+1}-p_{k}-n_{k},
$$

where $\sigma_{1}=\min \left\{e^{c}, e^{1-60 \beta}\right\}$. Also, by adapting $\sigma_{1}>1$ if necessary, we have

$$
D_{n_{k}, n_{k+1}-1}(x) \geq \sigma_{1}^{n_{k+1}-n_{k}} .
$$

Given $n \geq 0$, let $n_{k} \leq n$ be the last return time of $x$. Then

$$
D_{n}(x)=D(x)\left(\prod_{j=1}^{k-1} D_{n_{j}, n_{j+1}-1}(x)\right) D_{n_{k}, n}(x),
$$

where if $n_{1}=0$ then $D(x)=1$ otherwise $D(x)=D_{n_{1}-1}(x)$. In the latter case, from (34) and (35), we have

$$
D_{j}(x) \geq \delta^{2} \sigma_{1}^{j} \quad \text { for all } 0 \leq j<n_{1},
$$

and also

$$
D_{n_{1}}(x) \geq \sigma_{1}^{n_{1}} .
$$

When $\theta<0$, we have $\left|f^{\prime}(x)\right|>-A_{1} \theta$, for all $x \in \mathbb{S}^{1}$ and so, in view of all the previous estimates, we can state that there exists a constant $\hat{c}=\hat{c}(\theta)>0$ such that, for every $j \geq 0$,

$$
D_{j}(x) \geq \hat{c} \sigma_{1}^{j} \quad \text { for all } x \in \mathbb{S}^{1} .
$$

Therefore, $f$ is a uniformly expanding map. This completes the proof.

Our goal is to prove that periodic points are expanding. In fact, we are able to prove more: for Lebesgue almost every point, including the periodic points, the Lyapunov exponent is positive.

PROPOSITION 7.2. For $a \in \Omega_{\theta}, \theta=0$, we have

$$
\lambda_{\text {inf }}(x)=\liminf _{n \rightarrow \infty} \frac{1}{n+1} \log \left|\left(f_{a}^{n}\right)^{\prime}(x)\right|>0,
$$

for Lebesgue almost every $x$ in $\mathbb{S}^{1}$, including the periodic points. 
Proof. When $\theta=0$ we have $D_{n}(a)=0$ for all $n \geq 0$. In this case, we are interested in estimating

$$
\lambda_{\text {inf }}(x)=\liminf _{n \rightarrow \infty} \frac{1}{n+1} \log D_{n}(x) .
$$

From (36) and estimates we carried out in the proof of the previous proposition, we only have to worry about the terms $D_{n_{k}}(x)$.

We put, for a positive constant $C \leq 100$,

$$
X_{k}=\left\{x \in \mathbb{S}^{1}:\left|f^{j}(x)-a\right| \geq e^{-C \beta j}, \text { for all } j \geq k\right\} .
$$

Let

$$
X=\bigcap_{n \geq 1} \bigcup_{k \geq n} X_{k}
$$

be the set of points whose orbits fail to satisfy the condition $\left|f^{j}(x)-a\right| \geq e^{-C \beta j}$ only a finite number of times. Clearly, $X$ contains all periodic points. From (2), for every $x \in X$ we have

$$
\left|f^{\prime}(x)\right| \geq e^{-2 C \beta j} \quad \text { for every } j \geq k
$$

Since

$$
\frac{1}{n_{k}+1} \log D_{n_{k}}(x) \geq \frac{n_{k}}{n_{k}+1}\left(\log \sigma_{1}+\frac{1}{n_{k}} \log \left|f^{\prime}\left(x_{n_{k}}\right)\right|\right),
$$

we realize that, if $x$ is not a pre-image of the critical point and

$$
\left|f^{\prime}\left(x_{n_{k}}\right)\right|>e^{-2 C \beta n_{k}}
$$

for all returns $n_{k}$ occurring after an iterate $L=L(x)$, then we will have

$$
\lambda_{\text {inf }}(x) \geq \log \sigma_{1}-2 C \beta .
$$

If $\beta$ is taken sufficiently small, e.g., $\beta<10^{-4}$, then $\lambda_{\text {inf }}(x)>0$.

In the sequel we only sketch the proof of why $X$ has full measure since the arguments are substantially similar to those used in $\S \S 3,4$ and 5 . Let us reconsider the partition of the phase space introduced in $\S 3$. At this time we write

$$
\mathcal{P}^{\prime}:=\left\{I_{m, k}: m \geq \Delta+1,1 \leq k \leq m^{2}\right\}
$$

and

$$
\mathcal{P}_{0}:=\left\{\mathbb{S}^{1} \backslash(a-\delta, a+\delta)\right\} \cup \mathcal{P}^{\prime} .
$$

Analogously to what was done for intervals of parameters (see $\S 4$ ) we proceed by considering for each $\omega \in \mathcal{P}_{0}$ its iterates $f^{n}(\omega)$. Then we define inductively a sequence of partitions $\left(\mathcal{P}_{n}\right)_{n}$ obtained as successive refinements of $\mathcal{P}_{0}$ using an analogous algorithm like that described in that section, although in this context we do not need to perform any exclusions. Adapting results presented in $\$ 5$ (namely Lemmas 5.2 and 5.4) it is straightforward to prove that up to time $n$ points in the same element $\omega \in \mathcal{P}_{n}$ have almost indistinguishable orbits and the derivatives along these orbits are comparable up to a constant $C_{1}$ (bounded distortion).

Now fix $k \in \mathbb{N}$ (large). Given $n \geq k$ we define

$$
X_{k, n}=\left\{x \in \mathbb{S}^{1}:\left|f^{j}(x)-a\right| \geq e^{-C \beta j}, k \leq j \leq n\right\}
$$


and

$$
\hat{X}_{k, n}=\bigcup_{\omega \in \mathcal{P}_{n}}\left\{x \in \omega: \omega \subset X_{k, n}\right\} .
$$

More precisely, if $\omega \in \mathcal{P}_{n-1}$ first we define a partition of $\omega$ induced by $\mathcal{P}_{0}$ on $f^{n}(\omega)$. At time $n$, a subinterval $\omega^{\prime}$ of $\omega$ which will not contribute for the set $\hat{X}_{k, n}$ must correspond to $U_{m}(a) \cap f^{n}(\omega)$ for some $m$ with $|m|>[C \beta]-1$. Then

$$
\frac{\operatorname{Leb}\left(\omega^{\prime}\right)}{\operatorname{Leb}(\omega)} \leq C_{1} \frac{\operatorname{Leb}\left(f^{n}\left(\omega^{\prime}\right)\right)}{\operatorname{Leb}\left(f^{n}(\omega)\right)} \leq 2 C_{1} \frac{e^{-C \beta}}{e^{-\beta / 2}},
$$

where the first inequality follows from the bounded distortion of derivatives of $f$ over orbits of points in the same element of $\mathcal{P}_{n}$ up to time $n$, and the second one is a (pessimistic) bound for the growth of $\omega$ under the action of iterates of $f$. This last estimate can be obtained with analogous arguments to those in the proof of Lemma 5.2, taking into account that the orbit of the critical point $a$ is expanding for all times. From (40) it is straightforward to get

$$
\operatorname{Leb}\left(\hat{X}_{k, n} \backslash \hat{X}_{k, n+1}\right) \leq e^{-\varepsilon(n+1)} \operatorname{Leb}\left(\hat{X}_{k, n}\right)
$$

and setting

$$
\hat{X}_{k, \infty}:=\bigcap_{n \geq k} \hat{X}_{k, n}
$$

we get

$$
\lim _{k \rightarrow \infty} \operatorname{Leb}\left(\hat{X}_{k, \infty}\right)=1 .
$$

Since $\hat{X}_{k, \infty} \subset X_{k}$ for all $k$ this concludes the proof of Proposition 7.2.

\section{Proof of Theorem $A$}

Given $a \in \Omega_{\theta}, \theta=0$, for every $n \in \mathbb{N}$, there exist $\omega \in \mathcal{P}_{0, n}$ such that $a \in \omega$. It follows from the construction that, for fixed $n$, the family of sets $\Omega_{\theta, n}$ varies continuously with $\theta$. Then, for $\varepsilon>0$ small and $n$ sufficiently large, there exists $\theta_{0}=\theta_{0}(n, \varepsilon)<0$ such that there are intervals of parameters $\omega_{\theta} \in \mathcal{P}_{\theta, n} \varepsilon$-close to $\omega$, for every $\theta_{0} \leq \theta \leq 0$. Since each partition $\mathcal{P}_{\theta, n+1}$ is obtained from $\mathcal{P}_{\theta, n}$ and $\Omega_{\theta}$ is constructed by successive refinement of such partitions, it follows that there are $a_{\theta} \in \Omega_{\theta}, \theta_{0}<\theta$ accumulating on $a$. Therefore, arbitrarily near $f_{a, 0}$ there is a map $f_{a_{\theta}, \theta}$, for some $a_{\theta} \in \Omega_{\theta}, \theta<0$. Thus, by Proposition 7.1, for every $a \in \Omega_{0}, f_{a, 0}$ is approximated by (uniformly) expanding maps. Since the presence of a critical point is an obstruction for expansiveness, one concludes that $f_{a, 0}$ belongs to the boundary of a uniformly expanding domain, proving part (1) of the statement.

The bifurcations constructed above are not of the (NH) type due to the recurrence control imposed while constructing $\Omega_{\theta}$ : if the orbit of a critical point $a$ returns too close to itself this parameter is excluded. Therefore, the critical point is non-periodic. Furthermore, from Proposition 7.2 we know that periodic orbits of $f_{a, 0}, a \in \Omega_{0}$, are uniformly expanding since their Lyapunov exponents are (uniformly) positive.

Moreover, there is a full measure subset $\mathcal{A}$ of $\Omega_{0}$ such that the corresponding bifurcations are not of the (NT) type: fixing an interval $I_{n}$ of radius $1 / n$ centered in the 
critical point, for $n$ large enough, the set of parameters $\mathcal{B}_{n}$ such that the critical orbit never goes into $I_{n}$ has zero measure.

Therefore, the set of parameters in $\Omega_{0}$ such that the critical orbit is pre-periodic is contained in the countable union of the sets $\mathcal{B}_{n}$ and has zero measure (recall that if an orbit is non-wandering then it cannot be pre-periodic). This proves part (2) and concludes the proof of Theorem A.

\section{Proof of Theorem $B$ (invariant measures)}

From now on we fix $\theta \leq 0$ and a parameter $a \in \Omega_{\theta}$. We are going to show how to construct an invariant and absolutely continuous measure for such a map. For simplicity we consider $f=f_{a, \theta}$.

We follow the strategy of inducing Lebesgue measures in small pieces of our phase space. Taking into account how much of this subject is classical, we restrict ourselves to stating the realm of conditions which yield a point where getting such a measure is a matter of applying very well-known arguments present in, e.g., [4].

First of all, let us reintroduce some terminology and notation in a slightly different way. We begin with a sequence of partitions in the phase space which will be denoted by $\left(\mathcal{Q}_{n}\right)_{n}$ and which resembles very closely the partition $\left(\mathcal{P}_{n}\right)_{n}$ in the parameter space introduced in $\S 3$. We define

$$
\mathcal{Q}_{0}=\left\{I_{m, k}|m \geq \Delta| 1 \leq k \leq m^{2}\right\},
$$

where $I_{m, k}$ is defined in exactly the same way as in $\S 3$.

Successive refinements on $\mathcal{Q}_{0}$ will be made by taking into account the dynamic of $f$.

Let $\lambda$ denote the Lebesgue measure normalized in $U_{\Delta}$ and consider the pull-back of $\lambda$ given by

$$
f_{*}^{j}(\lambda)(A)=\lambda\left(f^{-j}(A)\right)=\operatorname{Leb}\left(f^{-j}(A) \cap U_{\Delta}\right) / \operatorname{Leb}\left(U_{\Delta}\right) .
$$

We define

$$
\mu_{n}=\frac{1}{n} \sum_{j=0}^{n-1} f_{*}^{j}(\lambda) .
$$

For each $n$ we have that $\mu_{n}$ is a finite measure, absolutely continuous with respect to Lebesgue. Putting $\mu$ as any limit point of this sequence in the weak*-topology, it is easy to see that such a measure is invariant under $f$.

In order to establish that $\mu$ is absolutely continuous with respect to the Lebesgue measure, we follow the strategy in [4]: analyzing (41) we divide the dynamics of each $\omega$ taking into account the concepts of returns, bound periods and free iterates. We consider four sequences of measures $\left(\mathcal{X}_{n}\right),\left(\mathcal{Y}_{n}\right),\left(\mathcal{Z}_{n}\right)$ and $\left(\mathcal{W}_{n}\right)$ such that

$$
\mu_{n} \leq \frac{1}{n} \sum_{j=0}^{n-1}\left(\mathcal{X}_{n}+\mathcal{Y}_{n}+\mathcal{Z}_{n}+\mathcal{W}_{n}\right)
$$

The proof then relies on the statement that these four measures are uniformly bounded in $L^{1}$-norm. To accomplish this we need two main results. The first one is a complete control of the distortion while iterating the intervals $\omega$ (Lemma 1.5 in [4]). Its contents are presented in the next lemma. 
LEMMA 9.1. Let $\omega$ be an interval on $\mathbb{S}^{1}$ whose points have the same dynamical evolution up to time n: the same returns, host intervals, and bound and free periods. Then we have

$$
\left|\frac{\left(f^{n}\right)^{\prime}(x)}{\left(f^{n}\right)^{\prime}(y)}\right| \leq C \quad \text { for some constant } C \text { independent of } n \text { and } \omega .
$$

The proof of this lemma relies on the same estimates we have already established in the preceding sections and so we will omit it. (See Lemma 5.1 and [4].)

The second auxiliary result we need is also classical and can be stated as follows: the probability of an orbit to avoid a fixed region around the criticality decreases exponentially fast. More precisely, we have the next lemma.

LEMMA 9.2. Let $\epsilon>0$ be sufficiently small. Then there is a constant $C>0$ and $\eta_{1}>0$ such that

$$
\operatorname{Leb}\left\{x: f^{j}(x) \notin(a-\epsilon, a+\epsilon), j=1, \ldots, k\right\} \leq C \eta_{1}^{k} .
$$

For the proof, see [4, Sublemma 3.6].

\section{Proof of Theorem $C$}

For each pair $(a, \theta)$ let us denote by $p_{a, \theta}$ the analytical continuation of the fixed point $p$ of $f$. The next lemma asserts that it is possible to extract from each two-parameter family $\left(f_{a, \theta}\right)_{a, \theta}$ a one-parameter family $\left(f_{a, \theta}\right)_{\theta}$ whose members have their critical point mapped onto its fixed point $p_{a, \theta}$.

LEMMA 10.1. For every $\left(f_{a, \theta}\right)_{a, \theta} \in \mathcal{U}$ there exists a one-parameter family $f_{\bar{a}(\theta), \theta}(x)$ such that $\bar{a}(\cdot)$ is a $C^{2}$-map defined for $\theta \leq 0$ and such that $f_{\bar{a}(\theta), \theta}(\bar{a}(\theta))=p_{\bar{a}(\theta), \theta}$. Moreover, there exists $\epsilon>0$ such that $f_{a, \theta}(a) \neq p_{a, \theta}$ for every $0<|a-\bar{a}(\theta)|<\epsilon$.

Proof. Let us define

$$
H(a, \theta)=f_{a, \theta}\left(f_{a, \theta}(a)\right)-f_{a, \theta}(a) .
$$

For $a=\bar{a}$ and $\theta=-1$ we have $H(a, \theta)=0$. Moreover,

$$
\begin{aligned}
\partial_{a} H(a, \theta)= & \partial_{a}\left(f_{a, \theta}(f(a, \theta, a))-f_{a, \theta}(a)\right) \\
= & \partial_{a} f_{a, \theta}\left(f_{a, \theta}(a)\right)+\partial_{x} f_{a, \theta}\left(f_{a, \theta}(a)\right)\left[\partial_{a} f_{a, \theta}(a)+\partial_{x} f_{a, \theta}(a)\right] \\
& -\partial_{a} f_{a, \theta}(a)-\partial_{x} f_{a, \theta}(a) \\
= & \partial_{a} f_{a, \theta}\left(f_{a, \theta}(a)\right)+\left[\partial_{x} f_{a, \theta}\left(f_{a, \theta}(a)\right)-1\right]\left[\partial_{x} f_{a, \theta}(a)+\partial_{a} f_{a, \theta}(a)\right] .
\end{aligned}
$$

By assumption $\left(\mathrm{H}_{4}\right)$, for every family $\left(f_{a, \theta}\right)$ sufficiently close to $\left(\tilde{f}_{a, \theta}\right)$, we can suppose $\partial_{a} f_{a, \theta}\left(f_{a, \theta}(a)\right)>0$. From $\left(\mathrm{H}_{4}\right)$ and the local form (2), for all $\theta \leq 0$, we have $\partial_{x} f_{a, \theta}(a)+\partial_{a} f_{a, \theta}(a)>0$. Assumptions $\left(\mathrm{H}_{2}\right)$ and $\left(\mathrm{H}_{4}\right)$ imply that $\partial_{x} f_{a, \theta}\left(f_{a, \theta}(a)\right)-1>$ 0 . Therefore,

$$
\partial_{a} H(a, \theta)>0, \text { for every }(a, \theta) .
$$

By the implicit function theorem there exists a function $\bar{a}(\theta)$ such that for all $\theta$ close to -1 we have $H(\bar{a}(\theta), \theta)=0$. It is not difficult to see that we can extend $\bar{a}(\theta)$ for every $\theta \leq 0$. Moreover, the elements of this family are unique with this property in a neighborhood of $\bar{a}(\theta)$. This proves the lemma. 
Proof of Theorem $C$. Given $\left(f_{a, \theta}\right)_{a, \theta} \in \mathcal{U}$ by Lemma 10.1 we can reparametrize the family in such a way that, for some $\bar{a}$,

$$
f_{\bar{a}, \theta}(\bar{a})=p_{\bar{a}, \theta}
$$

for every $\theta \leq 0$. Clearly, $f_{\bar{a}, \theta}$ is uniformly expanding for all $\theta<0$.

For each $\theta \leq 0$, let $p_{1}, \ldots, p_{d-1}, d=\operatorname{deg}(f)$, be the distinctive pre-images of the fixed point $p_{\bar{a}}=p_{\bar{a}, \theta}$ by $f_{\bar{a}}=f_{\bar{a}, \theta}$ ordered by clockwise orientation. Let $I^{+}$and $I^{-}$be the arcs with endpoints $p_{\bar{a}}$ and $p_{1}$, and $p_{d-1}$ and $p_{\bar{a}}$, respectively. Let $\bar{a}_{j}^{+}$be the pre-images of $p_{\bar{a}}$ by an inverse branch of $f_{\bar{a}}^{j}$ in $I^{+}$. Since $f_{\bar{a}}$ restricted to $I^{+}$is strictly increasing and there are no fixed points of $f_{\bar{a}}$ in $I^{+}$, then the sequence $\left(\bar{a}_{j}^{+}\right)_{j}$ is decreasing and converges to $p_{\bar{a}}$. Analogously we define an increasing sequence $\bar{a}_{j}^{-}$converging to $p_{\bar{a}}$. The points $\bar{a}_{j}^{ \pm}$determine a partition of a neighborhood of $p_{\bar{a}}$ by intervals $\left(\bar{a}_{k+1}^{+}, \bar{a}_{k}^{+}\right]$and $\left[\bar{a}_{k}^{-}, \bar{a}_{k+1}^{-}\right)$. Thus we can induce a partition $I_{k}^{ \pm}$around $\bar{a}$ such that

$$
\xi\left(I_{k}^{+}\right)=f_{\bar{a}}\left(I_{k}^{+}\right)=\left(\bar{a}_{k}^{+}, \bar{a}_{k-1}^{+}\right] \text {and } \xi\left(I_{k}^{-}\right)=\left[\bar{a}_{k-1}^{-}, \bar{a}_{k}^{-}\right) .
$$

Moreover, by construction, we have

$$
\xi\left(\left(\bar{a}_{k}^{+}, \bar{a}_{k-1}^{+}\right]\right)=\left(\bar{a}_{k-1}^{+}, \bar{a}_{k-2}^{+}\right] \quad \text { and } \xi\left(\left[\bar{a}_{k-1}^{-}, \bar{a}_{k}^{-}\right)\right)=\left[\bar{a}_{k-2}^{-}, \bar{a}_{k-1}^{-}\right) .
$$

Hence,

$$
\xi^{k+1}\left(I_{k}^{+}\right)=\mathbb{S}^{1} \supset I_{k}^{+} \quad \text { and } \quad \xi^{j}\left(I_{k}^{+}\right) \subset I^{+} \cap I_{k}^{+}=\emptyset,
$$

for every $1 \leq j \leq k$. The same holds on changing the sign + to - . Therefore, for every $k \geq 1$ there exists a fixed point for $\xi^{k+1}$ inside $I_{+}^{k}$, that is, there exists a sequence $\left(a_{k}\right)_{k}$, $a_{k} \in I_{k-1}^{+}, k>2$, such that

$$
f_{a_{k}}^{k}\left(a_{k}\right)=\xi^{k}\left(a_{k}\right)=a_{k} .
$$

By construction, the orbit of $a_{k}=a_{k}(\theta)$ has a unique iterate inside the perturbation region $I_{0}$, where the derivative of $f_{a_{k}, \theta}$ decreases with $\theta$, and $k-1$ iterates outside $I_{0}$ where its derivative is almost the same for all $\theta$. Then, for some $\tilde{\theta}\left(a_{k}\right)$ sufficiently small, $a_{k}$ is a periodic sink for $f_{a_{k}, \tilde{\theta}}$. Fixing that $a_{k}$, the family $\left(f_{a_{k}, \theta}\right)_{\theta}$ starts inside the set of uniformly expanding maps and crosses the boundary of this set in some $\theta<\tilde{\theta}$. This proves Theorem C.

Acknowledgements. We are grateful to M. Viana for suggesting this subject to us and for several useful conversations and incentives. Besides our institutions we are grateful for the hospitality of IMPA where a major part of this work was done. This work was partially supported by CAPES, FAPESP (02/06531-6 and 03/03107-9), IM/AGIMB Brazil and PRONEX.

\section{REFERENCES}

[1] A. Avila and C. G. Moreira. Statistical properties of unimodal maps: smooth families with negative Schwarzian derivative. Geometric Methods in Dynamics. I. Astérisque 286 (2003), 81-118.

[2] M. Benedicks and L. Carleson. On iterations of $1-a x^{2}$ on (-1, 1). Ann. of Math. 122 (1985), 1-25. 
[3] M. Benedicks and L. Carleson. The dynamics of the Hénon map. Ann. of Math. 133 (1991), 73-169.

[4] M. Benedicks and L.-S. Young. Absolutely continuous invariant measures and random perturbations for certain one-dimensional maps. Ergod. Th. \& Dynam. Sys. 12 (1992), 13-37.

[5] Ch. Bonatti, L. J. Díaz and F. Vuillemin. Cubic tangencies and hyperbolic diffeomorphisms. Bol. Soc. Brasil. Mat. (N.S.) 29(1) (1998), 99-144.

[6] M. J. Costa. Saddle-node horseshoes giving rise to global hénon-like attractors. Anais Acad. Bras. Ciências 70 (1998), 393-400.

[7] J. Graczyk and G. Swiatek. Generic hyperbolicity in the logistic family. Ann. of Math. 146 (1997), 1-52.

[8] M. Jakobson. Absolutely continuous invariant measures for one-parameter families of one-dimensional maps. Comm. Math. Phys. 81 (1981), 39-88.

[9] S. Luzzatto and W. Tucker. Non-uniformly expanding dynamics in maps with singularities and criticalities. Publ. Math. Inst. Hautes Études Sci. 89 (2000), 179-226.

[10] S. Luzzatto and M. Viana. Positive Lyapunov exponents for Lorenz-like families with criticalities. Géométrie complexe et systèmes dynamiques (Orsay, 1995). Astérisque 261 (2000), 201-237.

[11] M. Lyubich. Dynamics of quadratic polynomials. I, II. Acta Math. 178(2) (1997), 185-247, $247-297$.

[12] M. Lyubich. Almost every real quadratic map is either regular or stochastic. Ann. of Math. (2) 156(1) (2002), 1-78.

[13] L. Mora and M. Viana. Abundance of strange attractors. Acta Math. 171(1) (1993), 1-71.

[14] C. G. Moreira and J.-C. Yoccoz. Stable intersections of regular Cantor sets with large Hausdorff dimensions. Ann. of Math. (2) 154(1) (2001), 45-96.

[15] F. J. Moreira. Cahotic dynamics of quadratic maps. Master's Thesis, Univ. Porto, 1992.

[16] S. Newhouse and J. Palis. Bifurcations of Morse-Smale dynamical systems. Dynamical Systems (Proc. Symp., University of Bahia, Salvador, 1971). Academic Press, New York, 1973, pp. 303-366.

[17] S. Newhouse and J. Palis. Cycles and bifurcation theory. Astérisque 31 (1976), 44-140.

[18] S. Newhouse, J. Palis and F. Takens. Stable arcs of diffeomorphisms. Bull. Amer. Math. Soc. 82(3) (1976), 499-502.

[19] S. Newhouse, J. Palis and F. Takens. Bifurcations and stability of families of diffeomorphisms. Publ. Math. Inst. Hautes Etudes Sci. 57 (1983), 5-71.

[20] J. Palis and F. Takens. Cycles and measure of bifurcation sets for two-dimensional diffeomorphisms. Invent. Math. 82 (1985), 397-422.

[21] J. Palis and F. Takens. Hyperbolicity and the creation of homoclinic orbits. Ann. of Math. 125 (1987), 337-374.

[22] J. Palis and M. Viana. High dimension diffeomorphisms displaying infinitely many periodic attractors. Ann. of Math. 140(1) (1994), 207-250.

[23] J. Palis and J.-C. Yoccoz. Homoclinic tangencies for hyperbolic sets of large Hausdorff dimension. Acta Math. 172 (1994), 91-136.

[24] I. L. Rios. Unfolding homoclinic tangencies inside horseshoes: hyperbolicity, fractal dimensions and persistent tangencies. Nonlinearity 14(3) (2001), 431-462.

[25] P. R. Sabini. Non-periodic bifurcations at the boundary of hyperbolic maps. PhD Thesis, IMPA, 2001.

[26] S. Smale. Differentiable dynamical systems. Bull. Am. Math. Soc. 73 (1967), 747-817.

[27] J. Sotomayor. Generic bifurcations of dynamical systems. Dynamical Systems (Proc. Symp., University of Bahia, Salvador, 1971). Academic Press, New York, 1973, pp. 561-582. 